\title{
Interaction of a planar reacting shock wave with an isotropic turbulent vorticity field
}

\author{
César Huete, ${ }^{1, *}$ Tai Jin, ${ }^{2,3, \dagger}$ Daniel Martínez-Ruiz, ${ }^{1,4}$ and Kun Luo ${ }^{2}$ \\ ${ }^{1}$ Grupo de Mecánica de Fluidos, Universidad Carlos III de Madrid, Leganés, 28911, Spain \\ ${ }^{2}$ College of Energy Engineering, Zhejiang University, 310027 Hangzhou, People's Republic of China \\ ${ }^{3}$ Department of Mechanical Engineering, University College London, WC1E7JE, London, United Kingdom \\ ${ }^{4}$ Aix-Marseille Université, IRPHE, 13384 Marseille, France \\ (Received 28 March 2017; revised manuscript received 26 September 2017; published 9 November 2017)
}

\begin{abstract}
Linear interaction analysis (LIA) is employed to investigate the interaction of reactive and nonreactive shock waves with isotropic vortical turbulence. The analysis is carried out, through Laplace-transform technique, accounting for long-time effects of vortical disturbances on the burnt-gas flow in the fast-reaction limit, where the reaction-region thickness is significantly small in comparison with the most representative turbulent length scales. Results provided by the opposite slow-reaction limit are also recollected. The reactive case is here restricted to situations where the overdriven detonation front does not exhibit self-induced oscillations nor inherent instabilities. The interaction of the planar detonation with a monochromatic pattern of perturbations is addressed first, and then a Fourier superposition for three-dimensional isotropic turbulent fields is employed to provide integral formulas for the amplification of the kinetic energy, enstrophy, and anisotropy downstream. Transitory evolution is also provided for single-frequency disturbances. In addition, further effects associated to the reaction rate, which have not been included in LIA, are studied through direct numerical simulations. The numerical computations, based on WENO-BO4-type scheme, provide spatial profiles of the turbulent structures downstream for four different conditions that include nonreacting shock waves, unstable reacting shock (sufficiently high activation energy), and stable reacting shocks for different detonation thicknesses. Effects of the propagation Mach number, chemical heat release, and burn rate are analyzed.
\end{abstract}

DOI: 10.1103/PhysRevE.96.053104

\section{INTRODUCTION}

The interaction of reactive and nonreactive shock waves with nonuniform flows is of practical interest for many areas that include hypersonic flight [1-3], propulsion [4-8], and astrophysics [9-11]. Although the effect of the shock passage has been studied extensively by theoretical, numerical, and experimental methods (see Refs. [1-3,12-26] and the additional literature entries found therein), a precise understanding of the physical mechanisms that modify the turbulent flow has not yet been accomplished due to the wide range of temporal and spatial scales involved and the effect of the sudden transformations of the aerothermal fluid properties. The reactive case, however, has received considerably less attention, in part due to the inherent complexities involved in the combustion-process modeling [27-33].

In continuous detonation engines, such as shcramjets and pulse detonation engines, the propulsion device must confront adversities related to the high flow velocity that limits the mixing time between the reactant and oxygen. As the residence time of the reactants in the combustor is short in supersonic regimes, ignition typically cannot be achieved by relying on diffusion and heat conduction alone [6]. Shock waves positively contribute to ignite the mixture through the inherent temperature rise across the shock wave, and mixing enhancement by the turbulence amplification across the shock [4,5]. The critical conditions for shock-induced ignition, which may later lead to an anchored detonation wave as the main mechanism for sustained combustion, were studied in [34]

\footnotetext{
*chuete@ing.uc3m.es

†tai.jin@ucl.ac.uk
}

for a laminar configuration adopting a simplified one-step irreversible reaction in the large-activation-energy limit. Even if satisfactorily formed, the detonation wave must prove stable against turbulent fluctuations that are conveyed by the incoming air and amplified through the previous shock waves.

In addition to propulsion applications, the interaction of detonation waves with nonuniform patterns also is of interest in astrophysics, where ionization fronts resemble combustion waves in many respects, with the so-called R-type fronts being qualitatively similar to strong chemical detonations [9]. For example, regular shock-turbulence-interaction models, as that one proposed here, have been used to characterize the interaction of strong shocks arising from core-collapse supernovae with turbulence originated by the nuclear shell burning $[10,11]$. Dissociation effects, affecting the energy balance across the shock, modify the total mass compression ratio and then the postshock turbulent flow properties. Similar analogies are found in inertial confinement fusion, where strong shocks travel through a nonuniform deuteriumfilled $\mathrm{CH}$ foam, triggering ionization or radiation of thermal energy [35].

For nonreactive shock waves, linear interaction analysis (LIA) predictions have been found to be consistent with direct numerical simulation (DNS) results, with the latter converging to the former as the turbulent Mach number and the shock thickness become sufficiently small, even for low Taylor-microscale Reynolds numbers $\operatorname{Re}_{t}[22,23]$. For reactive shock waves, however, an analogous study that involves both DNS, capable of capturing the wide range of scales involved in the problem, and LIA has not yet been accomplished. As the parameters that would characterize the interaction of detonation waves with real turbulence flows are too numerous, a simplified version of the problem is conveniently selected 
in this work: the weak turbulent flow is assumed to be isotropic and incompressible (upstream density perturbations are disregarded) and the detonation wave is described with a one-step Arrhenius model for DNS analysis. For LIA, the detonation wave is taken as a pure discontinuity, as previously done in Refs. [27,28], although some results involving the opposite thick-detonation limit [32] are retrieved and commented. Thus, the governing nondimensional parameters that affect the turbulence amplification ratio for LIA reduce to the adiabatic gas index $\gamma$, the dimensionless heat release $q$, and the propagation Mach number $M_{0}$. DNS involve more potentially relevant parameters such as the detonation thickness $\ell$ (or, equivalently, the reaction rate) and the activation energy $E_{a}$.

The small-amplitude perturbation approach adopted in LIA is only applicable to situations where viscous and nonlinear effects are neglected across the detonation wave, then constraining the applicability of the model to weak turbulent perturbations and stable detonation waves. In this sense, realistic LIA results are expected for sufficiently overdriven detonations [36,37], with the Mach propagation number $M_{0}$ being significantly higher than the Chapman-Jouguet value $M_{\mathrm{CJ}}$. Previous LIA-based works relevant to this paper on reactive shocks can be cataloged based on the relative value of the detonation thickness to the characteristic turbulence length. The limit that assumes the induction layer to be much thinner than the most representative turbulent structures upstream was taken in Refs. [27,28,31], while the opposite limit, focused on small-scale perturbations, was studied in Ref. [32]. The LIA carried out in this paper, which pertains to the former configuration, employs the linearized Euler equations and the perturbed reacting Rankine-Hugoniot relationships. Focusing most directly on the results of greatest interest, asymptotic analysis is carried out to compute the long-time effects of vortical disturbances on the burnt-gas flow. Fourier superposition for three-dimensional isotropic turbulent fields is later employed to provide integral formulas for the amplification of the kinetic energy, enstrophy, and the degree of anisotropy in the downstream kinetic energy.

An exhaustive simulation of compressible turbulence requires numerical methods that simultaneously avoid excessive damping of spatial features over a large range of length scales and prevent spurious oscillations near shocks and shocklets through robust shock capturing. In this paper, a numerical code validated in Ref. [33], which uses a high-resolution bandwidthoptimized scheme (WENO-BO4) with eighth-order-accurate standard central differences, is employed to compute the reactive Navier-Stokes equations. The third-order TVD (total variation diminishing) Runge-Kutta multistage method is used for time integration. Numerical results are used to provide further insight on the detonation-turbulence phenomenon and to address configurations where LIA can not be applied. For example, with the aid of DNS, the impact of self-induced oscillations and the effect of the finite reaction rates in the downstream turbulent structures has been analyzed.

The paper begins by introducing the LIA formalism in Sec. II. The properties of the perturbation-free detonation wave are first shown. Then, the interaction with a single-mode vorticity field is addressed by integrating the Euler equations in the burnt gas. The long-time response of the detonation wave is analyzed and the far-field acoustic and rotational-entropic perturbations in the burnt-gas flow are characterized. The dependence of the amplitude of the perturbations with the interaction properties is provided explicitly. Section III shows the interaction of the reacting shock with three-dimensional isotropic vorticity fields. The study includes results obtained with the linear fast-reaction model and DNS. LIA provides integral formulas for the overall kinetic energy and vorticity amplification as a function of the adiabatic gas index $\gamma$, the relative heat release $q$, and the propagating Mach number $M_{0}$. Results rendered by the opposite slow-reaction limit are commented too. The degree of anisotropy in the downstream flow is investigated for both nonreactive and reactive shock waves. DNS are performed to display the detonation shape and the influence of the detonation parameters on the vorticity structures. The amplification of the streamwise and lateral Reynolds stresses through the detonation wave are computed, along with the total kinetic energy and the vorticity-amplification ratios. A discussion on both LIA and DNS is offered at the end of this section. Finally, concluding remarks are provided in Sec. IV. The transitory evolution of the planar detonation wave and the Laplace-transform formalism used to calculate it is shown in Appendix A. Details of the DNS are given in Appendix B.

\section{INTERACTION WITH A TWO-DIMENSIONAL MONOCHROMATIC VORTICITY FIELD}

\section{A. Steady, one-dimensional, ZND detonation front}

Following the seminal works of Zel'dovich [38], Neumann [39], and Döring [40] (ZND) developed independently in the early 1940s, the perturbation-free detonation structure is described as a nonreactive planar shock wave followed by a nonviscous diffusionless reactive layer. The evolution of the different flow variables across a steady planar detonation moving with velocity $D$ in a stagnant gaseous mixture is determined by the reacting Rankine-Hugoniot (RH) jump conditions. In particular, for a perfect gas with constant specific-heat ratio $\gamma$, the values of the density, pressure, velocity, and sound speed immediately behind the detonation, denoted by $\rho_{d}, p_{d}, U_{d}$, and $a_{d}=\left(\gamma p_{d} / \rho_{d}\right)^{1 / 2}$, can be expressed in terms of the detonation Mach number

$$
M_{0}=D / a_{0}
$$

in the form

$$
\begin{gathered}
R_{d}=\frac{\rho_{d}}{\rho_{0}}=\frac{D}{D-U_{d}}=\frac{(\gamma+1) M_{0}^{2}}{(\gamma-\kappa) M_{0}^{2}+1}, \\
P_{d}=\frac{p_{d}}{\rho_{0} D^{2}}=\frac{\gamma M_{0}^{2}(1+\kappa)+1}{\gamma(\gamma+1) M_{0}^{2}},
\end{gathered}
$$

and

$$
M_{d}=\frac{D-U_{d}}{a_{d}}=\sqrt{\frac{(\gamma-\kappa) M_{0}^{2}+1}{\gamma M_{0}^{2}(1+\kappa)+1}},
$$

where $\rho_{0}, p_{0}$, and $a_{0}=\left(\gamma p_{0} / \rho_{0}\right)^{1 / 2}$ represent the density, pressure, and sound speed in the unperturbed fresh mixture ahead of the detonation, and $M_{d}$ is the downstream Mach 

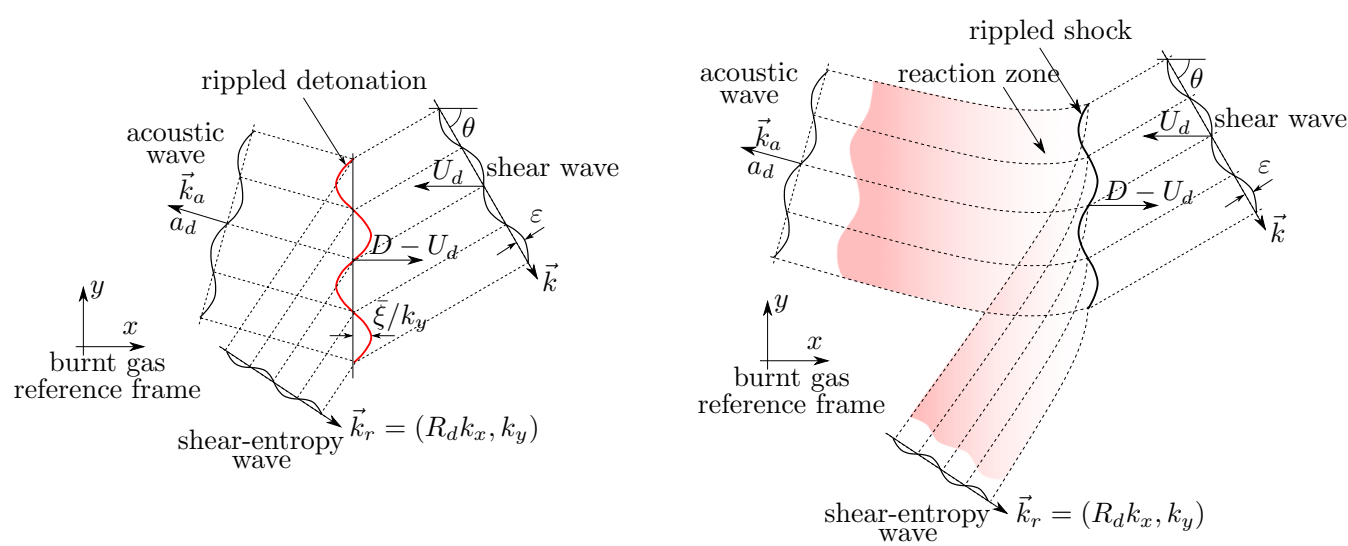

FIG. 1. Schematic view of a planar detonation wave interacting with a monochromatic shear wave in the fast-reaction limit [27] (left) and in the slow-reaction limit [32] (right).

number. The parameter

$$
\kappa=\left[\left(1-M_{0}^{-2}\right)^{2}-4 q M_{0}^{-2}\right]^{1 / 2}
$$

is a function of the detonation Mach number and of the dimensionless heat-release parameter $q=\left(\gamma^{2}-1\right) q^{\prime} /\left(2 a_{0}^{2}\right)$, with $q^{\prime}$ denoting the amount of chemical heat released in the detonation per unit mass of gas mixture. For detonations, the minimum stable propagation Mach number, determined by the sonic condition downstream $M_{d}=1$, is the ChapmanJouguet (CJ) value

$$
M_{\mathrm{CJ}}=(1+q)^{1 / 2}+q^{1 / 2},
$$

for which $\kappa=0$, whereas very strong detonations with $M_{0} \gg$ 1 correspond to $\kappa-1 \ll 1$. Reaction-zone development depends on the properties immediately behind the shock front. Those, the so-called Neumann values (identified with subscript $N)$, are obtained from (2)-(4) by using $\kappa=1-M_{0}^{-2}$, i.e., with $q=0$ in (5). Integration of the steady conservation equations, with $Y$ indicating the reaction progress variable $0 \leqslant Y \leqslant 1$ as reactants $(Y=1)$ proceed to products $(Y=0)$, yields

$$
\frac{p}{p_{\mathrm{N}}}=\frac{1+\gamma M_{\mathrm{N}}^{2}}{\gamma+1}+\frac{\gamma}{\gamma+1} \sqrt{\left(1-M_{\mathrm{N}}^{2}\right)^{2}-4 M_{\mathrm{N}}^{2} \frac{T_{0}}{T_{\mathrm{N}}} q(1-Y)}
$$

$$
\begin{aligned}
\frac{u}{u_{\mathrm{N}}}= & \frac{\rho_{\mathrm{N}}}{\rho}=\frac{1+\gamma M_{\mathrm{N}}^{2}}{M_{\mathrm{N}}^{2}(\gamma+1)}-\frac{1}{M_{\mathrm{N}}^{2}(\gamma+1)} \\
& \times \sqrt{\left(1-M_{\mathrm{N}}^{2}\right)^{2}-4 M_{\mathrm{N}}^{2} \frac{T_{0}}{T_{\mathrm{N}}} q(1-Y),}
\end{aligned}
$$

upon providing the shock boundary condition: $p=p_{\mathrm{N}}, u=$ $u_{\mathrm{N}}$, and $\rho=\rho_{\mathrm{N}}$ at $Y=1$. In a reference frame moving with the steady precursor shock wave, with $x_{s}$ indicating the distance from the shock, the spatial-dependent functions for $p, u$, and $\rho$ are obtained by simple integration of the conservation equation for the reaction progress variable

$$
\rho u \frac{d Y}{d x_{s}}=-r
$$

with

$$
r=B \rho Y e^{-T_{a} / T}
$$

being the reaction rate for one-step Arrhenius-type chemistry, $B$ referring to the pre-exponential factor, and $T_{a}$ describing the activation temperature, defined as the ratio of the activation energy to the gas constant $E_{a} / R_{g}$. The half-reaction length $\ell$, the distance measured from the shock front at which $Y=$ $\frac{1}{2}$, is taken as the characteristic detonation length, which is computed as

$$
\ell=\frac{a_{0}}{B} \frac{M_{0}}{R_{\mathrm{N}}} \int_{1}^{1 / 2} \frac{u}{u_{\mathrm{N}}} \exp \left(\beta \frac{T_{\mathrm{N}}}{T}\right) \frac{d Y}{Y},
$$

where $u / u_{\mathrm{N}}$ and $T / T_{\mathrm{N}}=\left(p / p_{\mathrm{N}}\right)\left(u / u_{\mathrm{N}}\right)$ are explicit functions of $Y$, previously provided in (7).

\section{B. Formulation of the linearized equations in the fast-reaction limit}

In the same manner as in Refs. [27,28,31], the dynamics of the detonation front excited by weak disturbances upstream will be described in the fast-reaction limit. In this limit (called by Korobeinikov [41] "detonation-wave model"), the detonation thickness $(\sim \ell)$ is much smaller than the characteristic upstream perturbation length $\lambda_{t}=2 \pi / k_{t}$, with $\lambda_{t}$ denoting the Taylor scale of the inflow turbulence and $k_{t}$ the corresponding wave number. As depicted in Fig. 1 (left), the detonation wave is then considered a discontinuity wave across which background and perturbation variables change, with the exclusion of the tangent velocity that remains invariable. The opposite limit sketched in Fig. 1 (right), corresponding to relatively small-size disturbances in comparison with the detonation thickness $\ell k_{t} \gg 1$, has been addressed in [32] and will not be considered here explicitly, although a parallel discussion will be carried out. In that limit corresponding to a relatively slow reaction velocity, disturbances are mainly governed by the inviscid weakly reactive Euler equations subject to the nonreacting Rankine-Hugoniot perturbation equations. Upon detonation and shock-induced distortion, a set of shear-entropy and acoustic waves is generated downstream, as sketched in Fig. 1 for the case of purely rotational perturbation upstream.

As explained in Chu and Kovásznay [42], the upstream and the downstream linear disturbances can be characterized in terms of acoustic, entropy, and vortical modes. The upstream monofrequency perturbation in the fresh-gas reference frame 
$\left(x_{0}, y\right)$ is determined by the divergence-free velocity perturbation wave, namely,

$$
\begin{aligned}
& \bar{u}_{0}\left(x_{0}, y\right)=\frac{u_{0}-\left\langle u_{0}\right\rangle}{\varepsilon\left\langle a_{d}\right\rangle}=\cos \left(k_{x} x_{0}\right) \cos \left(k_{y} y\right), \\
& \bar{v}_{0}\left(x_{0}, y\right)=\frac{v_{0}-\left\langle v_{0}\right\rangle}{\varepsilon\left\langle a_{d}\right\rangle}=\frac{k_{x}}{k_{y}} \sin \left(k_{x} x_{0}\right) \sin \left(k_{y} y\right),
\end{aligned}
$$

for the streamwise and crosswise perturbations, respectively. The brackets denote the time-averaged value of the flow variable, which is effectively null for the upstream velocity in the stagnant gas reference frame. The dimensionless factor $\varepsilon$ stands for the amplitude of the longitudinal velocity disturbances and $\vec{k}=\left(k_{x}, k_{y}\right)$ is the upstream wave-number vector. The associated nondimensional vorticity wave is

$$
\begin{aligned}
\bar{\omega}_{0}\left(x_{0}, y\right) & =\frac{1}{k_{y}}\left(\frac{\partial \bar{v}_{0}}{\partial x_{0}}-\frac{\partial \bar{u}_{0}}{\partial y}\right) \\
& =\left(1+\frac{k_{x}^{2}}{k_{y}^{2}}\right) \cos \left(k_{x} x_{0}\right) \sin \left(k_{y} y\right) .
\end{aligned}
$$

As a result of the interaction with the upstream vorticity field, the steady and planar front ripples and the burnt-gas fluid variables are affected consequently. The nondimensional value of the detonation position is $\varepsilon \bar{\xi}=k_{y}\left[x_{0, d}(t)-D t\right]$, as shown in Fig. 1. The dimensionless time is defined as $\tau=a_{d} k_{y} t$. Pressure, density, and velocity perturbations downstream, namely,

$$
\begin{aligned}
& \bar{p}=\frac{p-\left\langle p_{d}\right\rangle}{\varepsilon \gamma\left\langle p_{d}\right\rangle}, \quad \bar{\rho}=\frac{\rho-\left\langle\rho_{d}\right\rangle}{\varepsilon\left\langle\rho_{d}\right\rangle}, \\
& \bar{u}=\frac{u-\left\langle u_{d}\right\rangle}{\varepsilon\left\langle a_{d}\right\rangle}, \quad \text { and } \quad \bar{v}=\frac{v-\left\langle v_{d}\right\rangle}{\varepsilon\left\langle a_{d}\right\rangle}
\end{aligned}
$$

are employed to write the linear conservation equations in terms of dimensionless order-unity variables. Provided that $\bar{p}$ and $\bar{v}$ are always proportional to $\cos (y)$ and $\sin (y)$, and that Reynolds number is sufficiently high for the flow to be considered inviscid, the linear Euler equations read as

$$
\begin{array}{ll}
\frac{\partial \bar{\rho}}{\partial \tau}+\frac{\partial \bar{u}}{\partial\left(k_{y} x\right)}+\bar{v}=0, & \frac{\partial \bar{u}}{\partial \tau}+\frac{\partial \bar{p}}{\partial\left(k_{y} x\right)}=0, \\
\frac{\partial \bar{v}}{\partial \tau}-\bar{p}=0, & \frac{\partial \bar{p}}{\partial \tau}=\frac{\partial \bar{\rho}}{\partial \tau}
\end{array}
$$

for mass, $x$ momentum, $y$ momentum, and energy. They are rearranged to yield

$$
\frac{\partial^{2} \bar{p}}{\partial \tau^{2}}=\frac{\partial^{2} \bar{p}}{\partial\left(k_{y} x\right)^{2}}-\bar{p}
$$

as the two-dimensional periodically symmetric wave equation written in the burnt-gas reference frame $x=x_{0}-U_{d} t$.

The problem reduces to that of integrating (15) for $\tau \geqslant 0$ and within the domain delimited by the leading reflected sonic wave traveling backwards $k_{y} x=-\tau$ and the shock front moving upwards $k_{y} x=M_{d} \tau$. One boundary condition is provided by the isolated-detonation assumption, which translates into not considering the effect of the acoustic waves reaching the detonation front from behind. The other boundary condition is given by the linearized $\mathrm{RH}$ relationships

$$
\begin{aligned}
\frac{d \bar{\xi}}{d \tau} & =\frac{R_{d}\left(1-\Pi_{d}\right)}{2 M_{d}\left(R_{d}-1\right)} \bar{p}_{d}+\bar{u}_{0}, & \bar{u}_{d} & =\frac{1+\Pi_{d}}{2 M_{d}} \bar{p}_{d}+\bar{u}_{0}, \\
\bar{\rho}_{d} & =\frac{\Pi_{d}}{M_{d}^{2}} \bar{p}_{d}, & \bar{v}_{d} & =M_{d}\left(R_{d}-1\right) \bar{\xi}+\bar{v}_{0},
\end{aligned}
$$

where $\bar{p}_{d}, \bar{\rho}_{d}, \bar{u}_{d}$, and $\bar{v}_{d}$ are values of the nondimensional perturbations, defined in (13), right behind the detonation front. The equations involve the density ratio $R_{d}$ and the Mach number $M_{d}$ defined in (2) and (4), and the function

$$
\Pi_{d}=\frac{\left[1+M_{0}^{2}(1-\kappa)\right]^{2}}{4 M_{0}^{2}(1+q)}
$$

that accounts for the exothermicity effect in the perturbation equations, yielding $\Pi_{d}=M_{0}^{-2}$ for nonreactive shock waves.

The initial conditions are readily obtained by knowing that the detonation is initially planar, so that $\bar{\xi}=\bar{v}_{d}=0$. Correspondingly, the initial perturbations of pressure and streamwise velocity must satisfy $\bar{u}_{d}+\bar{p}_{d}=0$, as dictated by the first acoustic wave emitted backwards, thereby giving

$$
\bar{p}_{d_{i}}=-\frac{2 M_{d}}{1+\Pi_{d}+2 M_{d}}
$$

as the initial pressure perturbation at the detonation front.

\section{Evolution of the detonation front and the burnt-gas flow}

In absence of self-induced oscillations characteristic of unstable detonations, the isolated detonation front exhibits an asymptotic time-dependent response

$$
\bar{p}_{d}=\mathcal{P} \cos \left(\omega_{d} \tau+\phi_{p}\right),
$$

with $\omega_{d}=M_{d} R_{d} k_{x} / k_{y}$ indicating the nondimensional oscillation frequency and involving the order-unity function $\mathcal{P}$ and the phase shift $\phi_{p}$. It is convenient to define

$$
\zeta=\frac{\omega_{d}}{\left(1-M_{d}^{2}\right)^{1 / 2}}=\frac{R_{d} M_{d}}{\left(1-M_{d}^{2}\right)^{1 / 2}} \frac{k_{x}}{k_{y}}=\frac{R_{d} M_{d}}{\left(1-M_{d}^{2}\right)^{1 / 2}} \frac{1}{\tan \theta}
$$

as the characteristic oscillation frequency, with $\theta$ being the angle between the incident wave number $\vec{k}$ and the direction of propagation of the unperturbed detonation (see Fig. 1)

The transient evolution and the asymptotic long-time response of the detonation can be derived by employing the Laplace-transform technique to the previously mentioned differential equations. Details of this analysis are shown in Appendix A, along with computations for the transient detonation behavior. However, the long-time effects that will be later used to compute the interaction with an isotropic spectrum are provided in this section.

The asymptotic behavior of the corrugated detonation can be inferred from the Laplace-transform expression provided in (A6), with the poles indicating the type of evolution of the perturbations, in the dispersion relationship

$$
\begin{aligned}
& {\left[2 M_{d}\left(1-M_{d}^{2}\right) s \sqrt{s^{2}+1}+\left(1+\Pi_{d}\right)\left(1-M_{d}^{2}\right) s^{2}\right.} \\
& \left.\quad+\left(1-\Pi_{d}\right) R_{d} M_{d}^{2}\right]\left(s^{2}+\zeta^{2}\right)=0
\end{aligned}
$$


where $s$ refers to the Laplace variable applied to the scaled temporal variable $r=\left(1-M_{d}^{2}\right)^{1 / 2} \tau$. In this case, as $\left(1+\Pi_{d}\right)(1-$ $\left.M_{d}^{2}\right) \geqslant\left(1-\Pi_{d}\right) R_{d} M_{d}^{2}$, the first product in (21) owns no poles, then indicating that the only source of asymptotic oscillations comes from the upstream disturbances through the frequency $\zeta$.

The residues of the Laplace transform (A6) provide the corresponding amplitudes right behind the reactive shock, with $\mathcal{P}$ taking the form of

$$
\mathcal{P}_{\mathrm{hf}}=\frac{-2 M_{d}^{2}\left(R_{d}-1\right)}{2 M_{d}\left(1-M_{d}^{2}\right) \zeta \sqrt{1-\zeta^{2}}+\left(1-M_{d}^{2}\right)\left(1+\Pi_{d}\right) \zeta^{2}-M_{d}^{2} R_{d}\left(1-\Pi_{d}\right)}\left(\frac{1-M_{d}^{2}}{R_{d} M_{d}^{2}} \zeta^{2}-1\right)
$$

in the high-frequency regime $(\zeta \geqslant 1)$ and the associated phase shift being null.

On the other hand, in the low-frequency regime $(\zeta \leqslant 1)$, the asymptotic pressure function is conveniently rewritten as $\bar{p}_{d}=\mathcal{P}_{\mathrm{lf}_{1}} \cos \left(\omega_{d} \tau\right)+\mathcal{P}_{\mathrm{lf}_{2}} \sin \left(\omega_{d} \tau\right)$, so that $\mathcal{P}_{\mathrm{lf}}=\left(\mathcal{P}_{\mathrm{lf}_{1}}^{2}+\mathcal{P}_{\mathrm{lf}_{2}}^{2}\right)^{1 / 2}$ in (19), with

$$
\mathcal{P}_{\mathrm{lf}_{1}}=\frac{-2 M_{d}^{2}\left(R_{d}-1\right)\left[\left(1-M_{d}^{2}\right)\left(1+\Pi_{d}\right) \zeta^{2}-M_{d}^{2} R_{d}\left(1-\Pi_{d}\right)\right]}{4 M_{d}^{2}\left(1-M_{d}^{2}\right)^{2} \zeta^{2}\left(1-\zeta^{2}\right)+\left[\left(1-M_{d}^{2}\right)\left(1+\Pi_{d}\right) \zeta^{2}-M_{d}^{2} R_{d}\left(1-\Pi_{d}\right)\right]^{2}}\left(\frac{1-M_{d}^{2}}{R_{d} M_{d}^{2}} \zeta^{2}-1\right)
$$

and

$$
\mathcal{P}_{\mathrm{lf}_{2}}=\frac{4 M_{d}^{2}\left(1-M_{d}^{2}\right)^{2} \zeta \sqrt{1-\zeta^{2}}}{4 M_{d}^{2}\left(1-M_{d}^{2}\right)^{2} \zeta^{2}\left(1-\zeta^{2}\right)+\left[\left(1-M_{d}^{2}\right)\left(1+\Pi_{d}\right) \zeta^{2}-M_{d}^{2} R_{d}\left(1-\Pi_{d}\right)\right]^{2}}\left(\frac{1-M_{d}^{2}}{R_{d} M_{d}^{2}} \zeta^{2}-1\right)
$$

being the two orthogonal contributions and $\phi_{p}=$ $\arctan \left(\mathcal{P}_{\mathrm{lf}_{2}} / \mathcal{P}_{\mathrm{lf}_{1}}\right)$ referring to the phase shift in (19).

Once the amplitude of the pressure perturbations is determined, the rest of the flow variables are subsequently derived. For instance, the detonation ripple, which asymptotically oscillates with the same frequency $\omega_{d}$, is expressed as $\bar{\xi}=\mathcal{J}_{\mathrm{lf}_{1}} \sin \left(\omega_{d} \tau\right)+\mathcal{J}_{\mathrm{lf}_{2}} \cos \left(\omega_{d} \tau\right)$ for $\zeta \leqslant 1$, and $\bar{\xi}=$ $\mathcal{J}_{\text {hf }} \sin \left(\omega_{d} \tau\right)$ for $\zeta \geqslant 1$, with the corresponding coefficients being obtained through the RH equations (16) as

$$
\begin{aligned}
& \mathcal{J}_{\mathrm{lf}_{1}}=\frac{R_{d}\left(1-\Pi_{d}\right)}{2 M_{d}\left(R_{d}-1\right)} \frac{1}{\omega_{d}} \mathcal{P}_{\mathrm{lf}_{1}}+\frac{1}{\omega_{d}}, \\
& \mathcal{J}_{\mathrm{lf}_{2}}=-\frac{R_{d}\left(1-\Pi_{d}\right)}{2 M_{d}\left(R_{d}-1\right)} \frac{1}{\omega_{d}} \mathcal{P}_{\mathrm{lf}_{2}}
\end{aligned}
$$

for the long-wavelength regime, and

$$
\mathcal{J}_{\mathrm{hf}}=\frac{R_{d}\left(1-\Pi_{d}\right)}{2 M_{d}\left(R_{d}-1\right)} \frac{1}{\omega_{d}} \mathcal{P}_{\mathrm{hf}}+\frac{1}{\omega_{d}}
$$

for the short-wavelength regime.

To compute the far-field perturbations behind the detonation front, it is convenient to write separately the contributions coming from the acoustic fluctuations (which are irrotational and isentropic) and the steady rotational-entropic perturbations (that are isobaric) according to

$$
\begin{aligned}
& \bar{u}(x, \tau)=\bar{u}_{a}(x, \tau)+\bar{u}_{r}(x), \quad \bar{\rho}(x, \tau)=\bar{\rho}_{a}(x, \tau)+\bar{\rho}_{e}(x), \\
& \bar{v}(x, \tau)=\bar{v}_{a}(x, \tau)+\bar{v}_{r}(x), \quad \bar{p}(x, \tau)=\bar{p}_{a}(x, \tau) .
\end{aligned}
$$

\section{Acoustic perturbations downstream}

The sonic perturbations satisfy the bidimensional wave equation (15) that yields the well-known adiabatic dispersion relationship $\omega_{a}^{2}=\left(k_{a} / k_{y}\right)^{2}+1$. The condition that the detonation remains isolated from downstream influences
$\omega_{d}=\omega_{a}-M_{d}\left(k_{a} / k_{y}\right)$ is used to obtain

$$
\begin{aligned}
& \frac{k_{a}}{k_{y}}=\frac{M_{d} \omega_{d}-\sqrt{\omega_{d}^{2}-1+M_{d}^{2}}}{1-M_{d}^{2}} \text { and } \\
& \omega_{a}=\frac{\omega_{d}-M_{d} \sqrt{\omega_{d}^{2}-1+M_{d}^{2}}}{1-M_{d}^{2}}
\end{aligned}
$$

as the dimensionless acoustic wave number and the associated frequency, respectively. It is readily seen that acoustic perturbations downstream behave differently when $\omega_{d}$ is lower or greater than $\left(1-M_{d}^{2}\right)^{1 / 2}$, being the latter the condition for stable acoustic radiation downstream, i.e., the one that provides real values in (28). In this manner, the downstream pressure perturbation field splits into two distinguished regimes, namely, $\zeta<1$ or slow frequency regime, where the amplitude of the acoustic perturbation exponentially decays with the distance from the detonation front, and $\zeta>1$ or high frequency regime, where the acoustic radiation travels in form of constant-amplitude waves. The critical value $\zeta=1$ then indicates the condition at which stable sonic perturbations move parallel to the detonation front. This breakdown is similar to that shown in Refs. [1,26,27], where subsonic and supersonic regimes for acoustic waves downstream are obtained upon reference frame transformation that yields a steady interaction with an oblique shock or detonation.

The stable-radiation condition is conveniently rewritten in terms of the incident shear angle $\theta$. Then, for values of $\tan \theta<$ $\tan \theta_{c r}=R_{d} M_{d} /\left(1-M_{d}^{2}\right)^{1 / 2}$ the oscillating detonation front emits constant-amplitude sonic waves. As pointed out in Fig. 5 of Ref. [27], the value of $\tan \theta_{c r}$ (our definition for $\theta$ is the complementary angle of that used in [27]) is found to reach a constant value for highly overdriven detonations, while it grows steeply for weakly overdriven detonations, reaching $\theta_{c r}=\pi / 2$ for $M_{0}=M_{\mathrm{CJ}}$. The corresponding analytical 
expressions for $\tan \theta_{c r}$ in both limits are, respectively,

$$
\begin{aligned}
\left.\tan \theta_{c r}\right|_{M_{0} \gg M_{\mathrm{CJ}}} & =\left(\frac{\gamma+1}{\gamma-1}\right)^{1 / 2} \text { and } \\
\left.\tan \theta_{c r}\right|_{M_{0}-M_{\mathrm{CJ}} \ll 1} & \propto\left(\frac{M_{0}}{M_{\mathrm{CJ}}}-1\right)^{-1 / 4} .
\end{aligned}
$$

The asymptotic far-field solution of the acoustic disturbances is written in terms of monofrequency functions, representing stable traveling fronts in the high-frequency domain only $(\zeta>1)$. Then, the functions for the sonic contributions of pressure, density, and velocity variables are

$$
\begin{aligned}
\bar{p} & =\bar{\rho}_{a}=\mathcal{P} \cos \left(\omega_{a} \tau-k_{a} x\right), \\
\bar{u}_{a} & =\mathcal{U}_{a} \cos \left(\omega_{a} \tau-k_{a} x\right), \\
\bar{v}_{a} & =\mathcal{V}_{a} \sin \left(\omega_{a} \tau-k_{a} x\right),
\end{aligned}
$$

respectively. The acoustic velocity-perturbation amplitudes are proportional to the pressure changes through $\mathcal{U}_{a}=$ $\mathcal{P}\left(k_{a} / k_{y}\right) / \omega_{a}$ and $\mathcal{V}_{a}=\mathcal{P} / \omega_{a}$, with $\mathcal{P}$ provided in (22). The value of the dimensionless wave number $k_{a} / k_{y}$ and frequency $\omega_{a}$ associated to the traveling acoustic waves can be found in (28). It is seen that $k_{a} / k_{y}$ can be either negative or positive, the former representing the sonic waves propagating downwards in the burnt-gas reference frame, and the latter denoting the waves moving upwards, although never catching up the detonation wave as dictated by the isolated-front boundary condition. The detonation oscillation frequency $\omega_{d}=1$ marks the standing acoustic wave regime, therefore separating the left-traveling solution $\omega_{d}>1$ from the right-traveling regime $\left(1-M_{d}^{2}\right)^{1 / 2}<\omega_{d}<1$ in the burnt-gas reference frame.

The dimensionless kinetic energy associated to the sonic perturbations is given by $1 / 2\left(\left|\bar{u}_{a}\right|^{2}+\left|\bar{v}_{a}\right|^{2}\right)=1 / 2 \mathcal{P}^{2}$, with the value of $\mathcal{P}$ computed in Fig. 2 as a function of the relative incident shear angle $\theta_{c r}-\theta$, in degrees, for different values of overdrive $M / M_{\mathrm{CJ}}$. The value of $\theta_{c r}$, corresponding to $\zeta=1$, shows little variation with the overdrive factor, yielding $64.52^{\circ}$, $65.45^{\circ}$, and $67.33^{\circ}$ for $M / M_{\mathrm{CJ}}=1.5,2$, and 5, respectively. The right-side panel corresponds to the stable detonation radiating condition $\zeta>1$, then representing the long-time value of the pressure perturbations amplitude downstream within the domain $\left(-\tau<k_{y} x \leqslant M_{d} \tau\right)$. The acoustic waves at the leading edge downstream $k_{y} x \sim-\tau$, however, convey
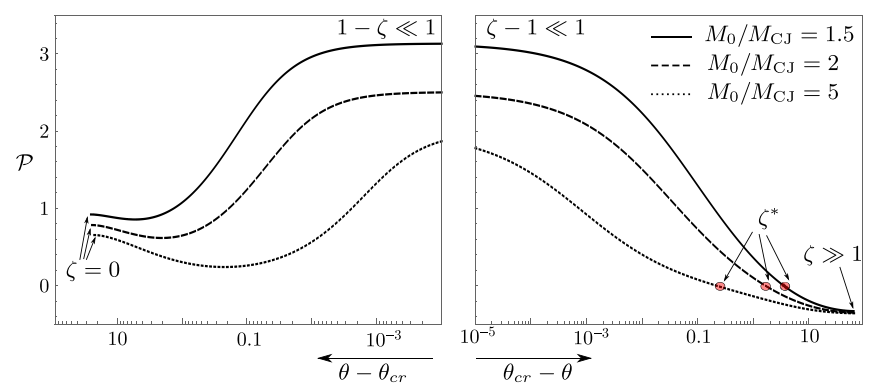

FIG. 2. Pressure perturbation $\mathcal{P}$ as a function of the relative incident shear angle $\theta_{c r}-\theta$ for $\zeta<1$ (left) and $\zeta>1$ (right). The gas properties are determined by $\gamma=1.4$ and $q=1$, and the overdrive degree is $M / M_{\mathrm{CJ}}=1.5,2$, and 5 . Red points represent the neutral transmission conditions. information of the transient evolution derived in Appendix A and computed in Fig. 16. There exists a neutral-transmission mode corresponding to $\mathcal{P}=0$, or $\zeta^{*}=M_{d} \sqrt{R_{d}} / \sqrt{1-M_{d}^{2}}>$ 1 as seen in (22). In this condition, the reacting shock oscillates with no generation of sound. For $\zeta>\zeta^{*}$ the phase reverses giving negative values for $\mathcal{P}$. The left-side panel corresponds to values for nonstable radiating condition $\zeta<1$, so this amplitude is only representative of pressure perturbations right behind the reacting front $x=M_{d} \tau$. It is readily observed that $\mathcal{P}$ decreases with the overdrive factor and that most of the acoustic kinetic energy is generated in a very narrow zone within the domain $\left|\theta_{c r}-\theta\right| \ll 1$ or $|\zeta-1| \ll 1$.

The acoustic disturbances stably radiated from the detonation wave $(\zeta>1)$ propagate with velocity $\vec{v}_{a}=a_{d} \hat{k}_{a}$ in the burnt-gas reference frame (see Fig. 1). The sonic wave points in direction

$$
\begin{aligned}
\hat{k}_{a} & =\left(\cos \theta_{a}, \sin \theta_{a}\right) \\
& =\left(\frac{M_{d} \zeta-\sqrt{\zeta^{2}-1}}{\zeta-M_{d} \sqrt{\zeta^{2}-1}}, \frac{\sqrt{1-M_{d}^{2}}}{\zeta-M_{d} \sqrt{\zeta^{2}-1}}\right)
\end{aligned}
$$

so that the sound energy flux $\vec{\Phi}$ carried by a planar acoustic wave in the system of coordinates in which the fluid is at rest is $\vec{\Phi}=a_{d} E_{s} \hat{k}_{a}$, with $E_{s}=\rho_{d} a_{d}^{2} \bar{p}^{2}$ accounting for the energy density of the sonic wave.

Written in the detonation-front reference frame, the normal to the sound wave is not aligned with the direction of propagation of energy $\vec{v}_{a}=a_{d}\left(\hat{k}_{a}-M_{d} \hat{x}\right)$ and the energy density is modified according to $\left(M_{d}-\cos \theta_{a}\right) E_{s}$. The streamwise component of the sound energy flux is conveniently scaled with the incident turbulent flux, namely, $1 / 2 \rho_{0} D\left(\left|u_{0}^{2}\right|+\left|v_{0}^{2}\right|\right)$, yielding

$$
\begin{aligned}
\phi_{x} & =\frac{\Phi_{x}}{1 / 2 \rho_{0} D\left(\left|u_{0}^{2}\right|+\left|v_{0}^{2}\right|\right)} \\
& =M_{d}^{-1}\left(1-M_{d} \cos \theta_{a}\right)\left(M_{d}-\cos \theta_{a}\right) \mathcal{P}^{2} \sin ^{2} \theta,
\end{aligned}
$$

with $\phi_{x}$ being a dimensionless order-unity quantity. The value of $\phi_{x}$ is computed in Fig. 3 as a function of the angle $\theta^{\prime}$ formed between streamwise component and the vector $\vec{v}_{a}$, which reads as

$$
\cos \theta^{\prime}=\frac{\cos \theta_{a}-M_{d}}{\sqrt{1+M_{d}^{2}-2 M_{d} \cos \theta_{a}}},
$$

there exhibiting two maxima (a two-wing shape in the polar inset similar to that shown in Ref. [18] for nonreactive shock waves). The plot has rotational symmetry around the $\hat{x}$ axis. The value of $\phi_{x}$ is zero for $\theta^{\prime}=\pi / 2$ and $\theta^{\prime}=\pi$, the former representing the radiation frontier $\zeta=1$ or $\theta_{a}=\cos ^{-1}\left(M_{d}\right)$, and the latter corresponding to the limit $\zeta \gg 1$ or $\theta_{a}-\pi \ll$ 1. The other value that produces no sound energy flux is determined by the condition $\mathcal{P}=0$, which corresponds to $\zeta=$ $\zeta^{*}$. This value, along with the angle of emission corresponding to the maximum energy flux in each wing, depends on the overdrive factor, as can be inferred in Fig. 3.

It was found in Ref. [18] that stronger shock waves generate more intense energy flux pointing backwards, while crosswise wings are barely affected by shock intensity. By way of 


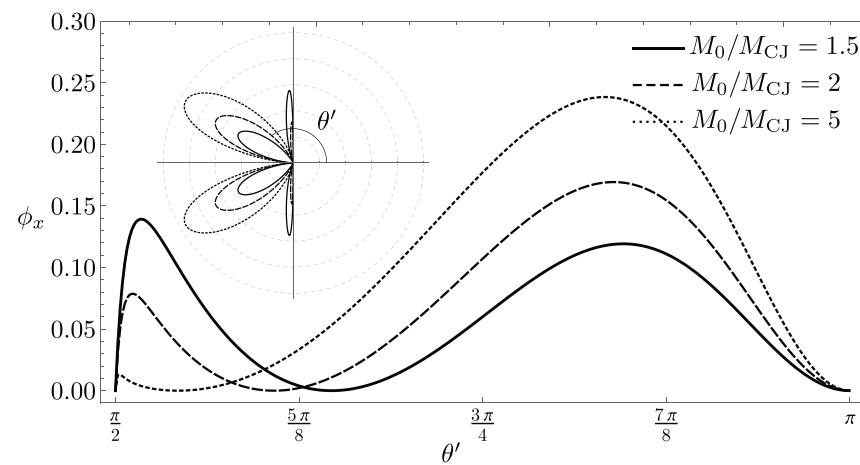

FIG. 3. Dimensionless acoustic energy flux $\phi_{x}$ as a function of the angle $\theta^{\prime}$. The gas properties are determined by $\gamma=1.4$ and $q=1$, and the overdrive degree is $M / M_{\mathrm{CJ}}=1.5,2$, and 5. The inset shows the same curves in a polar plot.

contrast, when heat release is considered, the amplitude of both quasistreamwise and crosswise wings are appreciably, and contrarily, affected by the overdrive. Higher overdrives are seen to produce noise whose intensity is mainly pointing backwards, while detonation with smaller overdrives tend to produce noise moving parallel to the detonation surface.

\section{Vortical-entropic perturbations in the burnt gas}

As viscous and heat transport processes can be neglected in first approximation for very large Reynolds numbers, the rotational-entropic disturbances generated behind the reacting shock are isentropically convected downstream with the fluid particles. The monochromatic functions

$$
\begin{aligned}
\bar{\rho}_{e} & =\mathcal{R}_{e} \cos \left(R_{d} k_{x} x+\phi_{e}\right), \\
\bar{u}_{r} & =\mathcal{U}_{r} \cos \left(R_{d} k_{x} x+\phi_{r}\right), \\
\bar{v}_{r} & =\mathcal{V}_{r} \sin \left(R_{d} k_{x} x+\phi_{r}\right)
\end{aligned}
$$

represent the spatial dependence of the far-field entropicrotational perturbations in the burnt-gas reference frame, with $\mathcal{R}_{e}$ being the amplitude of the isobaric density disturbances, and $\mathcal{U}_{r}$ and $\mathcal{V}_{r}$ being the amplitudes of the streamwise and transverse solenoidal velocity perturbations. The asymptotic monofrequency functions shown in (34) are computed in Fig. 18 along with the spatial distribution that accounts for the transient effects for $k_{y} x \sim 1$. It is there observed that the approaching time towards the asymptotic solution significantly depends on characteristic frequency.

The amplitude $\mathcal{R}_{e}$ is determined by the $\mathrm{RH}$ relationship upon acoustic contribution subtraction, yielding $\mathcal{R}_{e}=$ $\mathcal{P}\left(\Pi_{d}-M_{d}^{2}\right) / M_{d}^{2}$ in absence of upstream density perturbations. For upstream isentropic conditions, downstream density perturbations are all generated at the detonation front, then yielding $\phi_{e}=\phi_{p}=\arctan \left(\mathcal{P}_{\mathrm{lf}_{2}} / \mathcal{P}_{\mathrm{lf}_{1}}\right)$ for the associated phase shift. Similarly, temperature perturbations, obtained through the perfect gas equation of state, are produced by the perturbed detonation, yielding $\bar{T}_{a}=(\gamma-1) \bar{p}$ and $\bar{T}_{e}=\left(1-\Pi_{d} / M_{d}^{2}\right) \bar{p}$ for the acoustic and entropic contributions, respectively.
The amplitude of the longitudinal and transverse rotational velocity disturbances are

$$
\begin{aligned}
& \mathcal{U}_{r}=\frac{M_{d}^{2}}{M_{d}^{2}+\left(1-M_{d}^{2}\right) \zeta^{2}} \Omega \text { and } \\
& \mathcal{V}_{r}=\frac{\zeta M_{d} \sqrt{1-M_{d}^{2}}}{M_{d}^{2}+\left(1-M_{d}^{2}\right) \zeta^{2}} \Omega,
\end{aligned}
$$

respectively, with $\Omega$ indicating the amplitude of the dimensionless vorticity disturbances $\bar{\omega}=\left(\partial \bar{v}_{r}\right) /\left(\partial k_{y} x\right)-$ $\left(\partial \bar{u}_{r}\right) /\left(\partial k_{y} y\right)=g\left(k_{y} x\right) \sin \left(k_{y} y\right)$. Linear analysis, detailed in (A12), provides

$$
\begin{aligned}
g\left(k_{y} x\right) & =\Omega \cos \left(R_{d} k_{x} x+\phi_{r}\right) \\
& =\Omega_{1} \cos \left(R_{d} k_{x} x\right)+\Omega_{2} \bar{p}_{d}\left(\tau=k_{y} x / M_{d}\right)
\end{aligned}
$$

for the far-field dimensionless vorticity perturbations. As dictated by (36), there exist two sources for the vorticity disturbances in the burnt gas, namely, the direct amplification across the detonation wave due to the overall compression effect and the contribution due to the detonation front corrugation. The former, represented by the factor $\Omega_{1}=$ $R_{d}\left(1+k_{x}^{2} / k_{y}^{2}\right)=R_{d} / \sin ^{2} \theta$, is noticeably affected by the relative chemical energy as the value of the mass compression ratio $R_{d}$ increases with the overdrive, being $R_{\mathrm{N}}$ the maximum value for a given $M_{0}$. The latter, which includes the factor $\Omega_{2}=\left(R_{d}-1\right)\left(1-\Pi_{d}\right) /\left(2 M_{d}\right)$, is responsible of vorticity perturbations generated by the discontinuity front rippling. This factor, neglected in rapid distortion theory (RDT) [12], may interact constructively or destructively with $\Omega_{1}$ in forming the total vorticity perturbation amplitude $\Omega$. In particular, it is found that $\Omega=\Omega_{1}+\Omega_{2} \mathcal{P}_{\mathrm{hf}}$ for $\zeta \geqslant 1$, and $\Omega=\left(\Omega_{r}^{2}+\right.$ $\left.\Omega_{i}^{2}\right)^{1 / 2}$ for $\zeta \leqslant 1$, with $\Omega_{r}=\Omega_{1}+\Omega_{2} \mathcal{P}_{\mathrm{lf}_{1}}$ and $\Omega_{i}=\Omega_{2} \mathcal{P}_{\mathrm{lf}_{2}}$ representing the two orthogonal contributions. The associated rotational phase shift, null in the high-frequency regime, is $\phi_{r}=\Omega_{2} \mathcal{P}_{\mathrm{lf}_{2}} /\left(\Omega_{1}+\Omega_{2} \mathcal{P}_{\mathrm{lf}_{1}}\right)$ for $\zeta<1$.

Computations for $\Omega$ reveal that this factor diverges for values of $\theta \ll 1(\zeta \gg 1)$, as this limit corresponds to highly stretched solenoidal structures upstream that are further compressed downstream. It is therefore convenient to compute the actual vorticity amplification factor, that is $\left|\omega^{\prime}\right| /\left|\omega_{0}^{\prime}\right|=$ $\Omega \sin ^{2} \theta$, as shown in Fig. 4. It is also computed the factor $\Omega_{1} \sin ^{2} \theta=R_{d}$ as a measure of the compression ratio of the vortices when front-corrugation effects are neglected, as assumed in RDT. The curves show that intensity of the total vorticity field is generally smaller than the contribution predicted by RDT alone, except for very narrow zones close to the critical angle $\theta_{c r}(\zeta=1)$. This is in agreement with Ref. [12], where the authors found overestimated values for the turbulent amplification ratios, which are dominantly proportional to the value $\Omega$ shown in Fig. 4. In the low-frequency limit corresponding to $\theta \sim \pi / 2$ or $\zeta \ll 1$, the factor $\Omega$ approaches to unity, indicating that upstream vorticity perturbations are not modified by the detonation passage. On the other hand, the factor $\Omega$ tends to $\Omega_{1}$ in the high-frequency limit, indicating that detonation-rippling contribution is negligible. In neutraltransmission regime $\zeta=\zeta^{*}$, the vorticity field is only affected by overall one-dimensional compression $\Omega_{1} \sin ^{2} \theta=R_{d}$, as no pressure perturbations (nor entropic) are generated. The net 


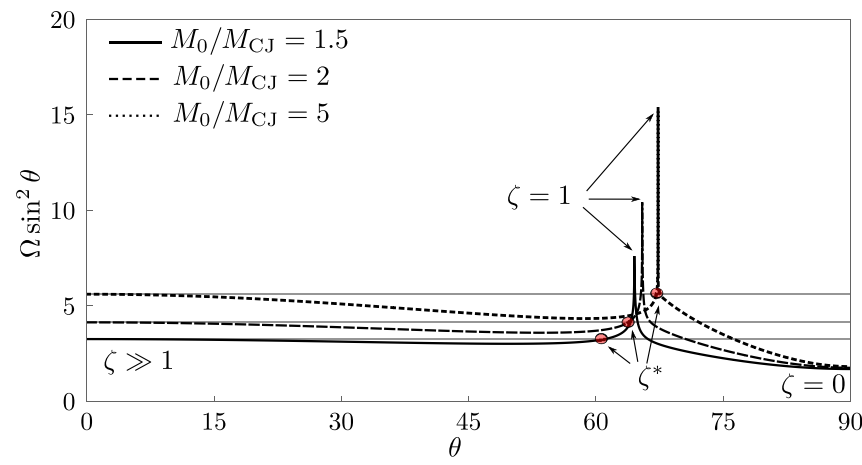

FIG. 4. Total vorticity amplification ratio $\Omega \sin ^{2} \theta$ (black lines) and $\Omega_{1} \sin ^{2} \theta=R_{d}$ (thin gray lines) as a function of the incident shear angle $\theta$. The gas properties are determined by $\gamma=1.4$ and $q=1$, and the overdrive degree is $M / M_{\mathrm{CJ}}=1.5,2$, and 5 . Red points represent the neutral transmission conditions.

effect of overdriving is seen to amplify the amplitude of the vorticity perturbations, an expected result that is in consonance with Fig. 6 in Ref. [27]. The effect of heat-release increase for a constant Mach number, then weakening of overdrive degree, is found to be in agreement with LIA results in the thick-reaction limit [32].

The longitudinal and transverse rotational velocity perturbations are used to define the rotational kinetic energy $1 / 2\left(\mathcal{U}_{r}^{2}+\mathcal{V}_{r}^{2}\right)$. The values of $\mathcal{U}_{r}$ and $\mathcal{V}_{r} \tan \theta$ are plotted in Fig. 5 as a function of the incident shear angle $\theta$ for $q=1, \gamma=1.4$, and different overdrive values $M_{0} / M_{\mathrm{CJ}}=$
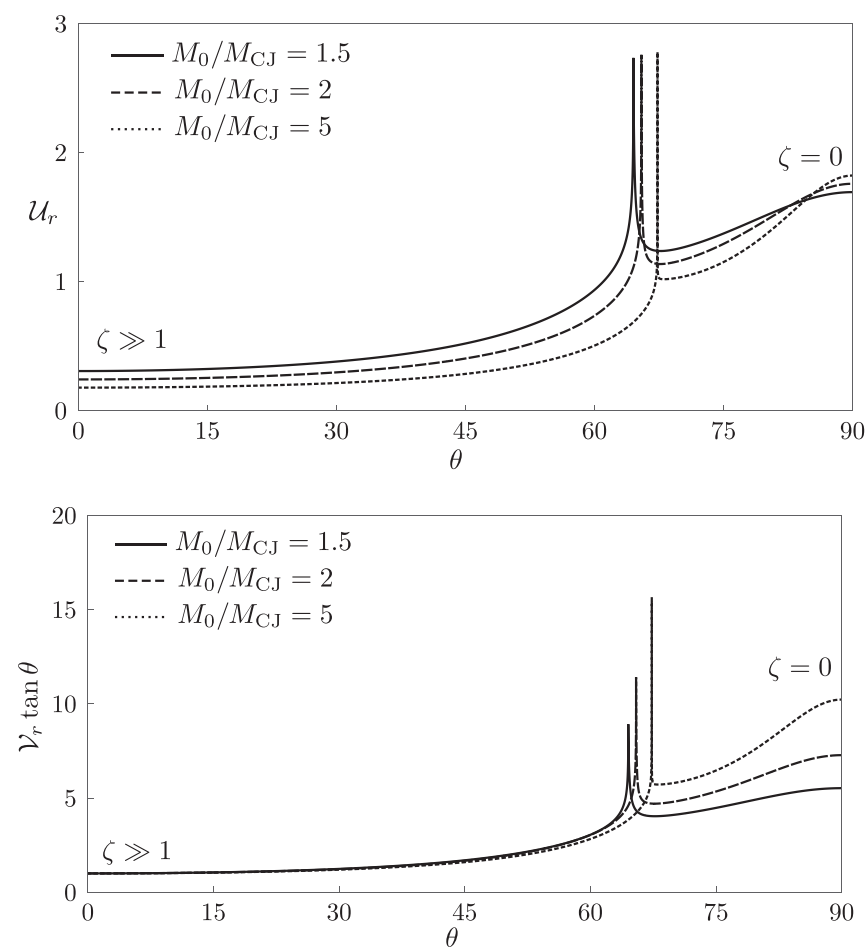

FIG. 5. Longitudinal (upper) and transverse (bottom) rotational velocity amplitudes $\mathcal{U}_{r}$ and $\mathcal{V}_{r} \tan \theta$, respectively, as a function of the incident shear angle $\theta$. The gas properties are determined by $\gamma=1.4$ and $q=1$, and the overdrive degree is $M / M_{\mathrm{CJ}}=1.5,2$, and 5 .
1.5, 2, and 5. Computations show that each of the amplitudes develops a pronounced finite-amplitude peak at the critical angle $\theta_{c r}$ corresponding to $\zeta=1$. The variation of the different amplitudes with $\theta$ is rapid in the near-transition region, in consonance with Fig. 2 . The amplitude $\mathcal{U}_{r}$ decreases with the overdrive intensity except for incident angles close to $\pi / 2$ $(\zeta \ll 1)$, where the value of $\mathcal{U}_{r}$ asymptotically approaches to

$$
\begin{aligned}
\left.\mathcal{U}_{r}\right|_{\zeta-1 \ll 1}= & \frac{(2+\gamma) M_{0}^{2}-1+\sqrt{M_{0}^{4}+1-2 M_{0}^{2}(1+2 q)}}{(\gamma+1) M_{0}^{2}} \\
& +O(\zeta) .
\end{aligned}
$$

That limit is representative of situations where the upstream perturbation field is dominated by highly stretched vortices in the longitudinal direction, with no or very little variation on the streamwise direction. On the other hand, the limit associated to vortices highly stretched in the crosswise direction yields $\left.\mathcal{U}_{r}\right|_{\zeta \gg 1}=R_{d}^{-1}+O\left(\zeta^{-1}\right)$ for the streamwise rotational velocity disturbances, indicating that longitudinal perturbation is only affected by the overall compression ratio $R_{d}$, as observed in Fig. 5. This is in agreement with Fig. 4 that shows that shock-corrugation effect does not play any role in downstream eddies setup for $\zeta \gg 1$, where the only contribution for the rotational field behind comes from the overall mass-compression effect.

Computations for $\mathcal{V}_{r}$ reveal that it approaches to 0 for $\zeta \ll 1$ and diverges for $\zeta \gg 1$ since the downstream perturbation amplitude $\left|v_{r}^{\prime}\right|$ is scaled with upstream longitudinal perturbation $\varepsilon$. It is therefore convenient to investigate the function $\left|v_{r}^{\prime}\right| /\left|v_{0}^{\prime}\right|=\mathcal{V}_{r} \tan \theta$ as a measure of the direct amplification factor, as shown in Fig. 5. The corresponding low-frequency limit for the transverse rotational contribution provides

$$
\begin{aligned}
\left.\mathcal{V}_{r} \tan \theta\right|_{\zeta-1 \ll 1} & =\frac{(2+\gamma) M_{0}^{2}-1+\sqrt{M_{0}^{4}+1-2 M_{0}^{2}(1+2 q)}}{1+\gamma M_{0}^{2}-\sqrt{M_{0}^{4}+1-2 M_{0}^{2}(1+2 q)}}+O(\zeta),
\end{aligned}
$$

while that corresponding to the high-frequency limit simply yields $\left.\mathcal{V}_{r} \tan \theta\right|_{\zeta \gg 1}=1+O\left(\zeta^{-1}\right)$. As expected, detonationrippling effect is negligible when $\zeta \gg 1$ so that vortices downstream are only affected by the wave-compression effect. The transverse contribution is found to remain unaltered after the detonation in this limit. It is important to remark that the limit representing high-frequency disturbances may arise from very small eddies upstream and/or very fast detonation waves, the former situation being possibly out of the limits of validity of the fast-reaction domain.

\section{INTERACTION WITH THREE-DIMENSIONAL ISOTROPIC VORTICITY FIELD}

\section{A. Turbulent kinetic energy}

As in previous works [31,32], the three-dimensional upstream flow is assumed to be homogeneous and isotropic, so the analysis is easily conducted by direct superposition of linear perturbations [43], whose amplitude has been computed in the previous section. Therefore, in this case, the 
amplitude of the incident shear wave $\varepsilon$ depends exclusively on the wave-number amplitude $|\vec{k}|=k$. The three-dimensional problem is conveniently formulated in spherical polar coordinates, so that the upstream velocity field holds $\left(\bar{u}_{0}, \bar{v}_{0}, \bar{w}_{0}\right)=$ $\varepsilon(\sin \theta \sin \varphi, \cos \theta \sin \varphi, \cos \varphi)$ and the associated wavenumber vector is $\vec{k}=k(-\sin \theta, \cos \theta \sin \varphi, \cos \theta \cos \varphi)$. The upstream mean-square disturbances are

$$
\begin{aligned}
& \left\langle\bar{u}_{0}^{2}\right\rangle=\int_{k^{3}}\left|\bar{u}_{0}\right|^{2} d k^{3}=\frac{8 \pi}{3} \int_{0}^{\infty} \varepsilon^{2}(k) k^{2} d k, \\
& \left\langle\bar{v}_{0}^{2}\right\rangle=\left\langle\bar{w}_{0}^{2}\right\rangle=\int_{k^{3}}\left|\bar{v}_{0}\right|^{2} d k^{3}=\frac{2 \pi}{3} \int_{0}^{\infty} \varepsilon^{2}(k) k^{2} d k
\end{aligned}
$$

and the corresponding turbulent kinetic energy (TKE) is

$$
\frac{1}{2}\left(\left\langle\bar{u}_{0}^{2}\right\rangle+\left\langle\bar{v}_{0}^{2}\right\rangle+\left\langle\bar{w}_{0}^{2}\right\rangle\right)=\frac{1}{2} \int_{0}^{\infty} E(k) d k,
$$

with $E(k)$ representing the three-dimensional energy spectrum, as provided in (B6) for DNS. Although it is true that post-detonation turbulence spectrum depends on the function $E(k)$, the kinetic energy amplification ratio does not as long as $E(k)$ is isotropic. After some straightforward algebra involving the reduction of the three-dimensional geometry into an equivalent two-dimensional case (see Ref. [18] for details), the amplification ratio across the detonation wave takes the form

$K=\frac{\left\langle\bar{u}^{2}\right\rangle+\left\langle\bar{v}^{2}\right\rangle+\left\langle\bar{w}^{2}\right\rangle}{\left\langle\bar{u}_{0}^{2}\right\rangle+\left\langle\bar{v}_{0}^{2}\right\rangle+\left\langle\bar{w}_{0}^{2}\right\rangle}=\frac{1}{2} \int_{0}^{\pi / 2}\left(\bar{u}^{2}+\bar{v}^{2}\right) \sin ^{3} \theta d \theta+\frac{1}{2}$.

As the far-field amplitude of the velocity perturbations is explicitly written as a function of the frequency $\zeta$, the normalized probability-density distribution

$$
\mathrm{P}(\zeta)=\frac{3}{2} \sin ^{3} \theta \frac{d \theta}{d \zeta}=\frac{3}{2} \frac{M_{d}^{4} R_{d}^{4} \sqrt{1-M_{d}^{2}}}{\left[M_{d}^{2} R_{d}^{2}+\zeta^{2}\left(1-M_{d}^{2}\right)\right]^{5 / 2}},
$$

satisfying $\int_{0}^{\infty} \mathrm{P}(\zeta) d \zeta=1$, is introduced to rewrite the kinetic energy as

$$
K=\frac{1}{3} \int_{0}^{\infty}\left(\bar{u}^{2}+\bar{v}^{2}\right) \mathrm{P}(\zeta) d \zeta+\frac{1}{2} .
$$

Equivalently, the amplification ratios for the longitudinal and transverse kinetic energy contributions can be computed with the aid of the probability density distribution. They are conveniently split into rotational and acoustic contributions, yielding

$$
L=L_{r}+L_{a}=\int_{0}^{\infty} \mathcal{U}_{r}^{2} \mathrm{P}(\zeta) d \zeta+\int_{1}^{\infty} \mathcal{U}_{a}^{2} \mathrm{P}(\zeta) d \zeta
$$

for the longitudinal part, and

$$
T=T_{r}+T_{a}=\frac{1}{2}\left[\int_{0}^{\infty} \mathcal{V}_{r}^{2} \mathrm{P}(\zeta) d \zeta+\int_{1}^{\infty} \mathcal{V}_{a}^{2} \mathrm{P}(\zeta) d \zeta+\frac{3}{2}\right]
$$

for the transverse contribution. The value of the amplitudes $\mathcal{U}_{r}$ and $\mathcal{V}_{r}$ are provided in (35) as a function of $\zeta$, and the acoustic amplitudes are $\mathcal{U}_{a}=\mathcal{P}\left(k_{a} / k_{y}\right) / \omega_{a}$ and $\mathcal{V}_{a}=\mathcal{P} / \omega_{a}$. It is straightforward to obtain the total turbulent kinetic

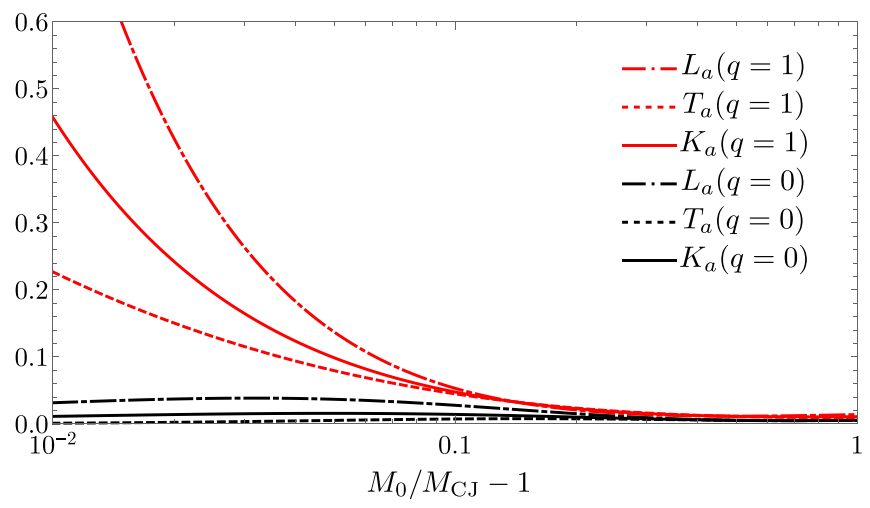

FIG. 6. $L_{a}$ (dotted-dashed line), $T_{a}$ (dashed line), and $K_{a}$ (solid line) as a function of the overdrive parameter $M_{0} / M_{\mathrm{CJ}}$ for $\gamma=1.4$, $q=1$ (red), and $q=0$ (black).

energy through $K=K_{r}+K_{a}$, with $K_{r}=\left(L_{r}+2 T_{r}\right) / 3$ and $K_{a}=\left(L_{a}+2 T_{a}\right) / 3$ representing the rotational and acoustic contributions, respectively.

The acoustic kinetic energy contribution, computed in Fig. 6 for $L_{a}$ (dotted line), $T_{a}$ (dashed line), and $K_{a}$ (solid line), is found to be relatively important uniquely for detonation regimes close to Chapman and Jouget. Both longitudinal and transverse components for the acoustic kinetic energy diverge, a token of the failure of linear theory in reproducing longtime responses in the limit $M_{0} / M_{\mathrm{CJ}}-1 \ll 1$. Computations also reveal that longitudinal contribution grows much faster when the overdrive factor approaches unity. It is seen that, for order-unity values of $M_{0} / M_{\mathrm{CJ}}-1$, acoustic kinetic energy is negligible when compared to rotational contribution, the latter being computed in Fig. 7. It has been found in [32] that sonic perturbations generated by sufficiently small-size perturbations are not affected by exothermicity, then providing an acoustic kinetic energy as that shown in Fig. 6 for $q=0$.

Figure 7 shows computations for $L_{r}$ (dotted-dashed line), $T_{r}$ (dashed line), and $K_{r}$ (solid line) for nonreactive $q=0$ (black) and reactive shock waves $q=1$ (red) traveling through diatomic gases, $\gamma=1.4$, as a function of the propagation Mach number for $M_{\mathrm{CJ}}<M_{0}<10$. The curves in black, which correspond to the canonical case of nonreacting shock waves propagating through an isotropic vorticity field, have been widely shown in literature since Ribner seminal work [2]. The arrows represent the effect of exothermicity in the limit of small-size rotational disturbances, according to Ref. [32]. To compute the total kinetic energy in the slow-reaction limit, the amplitude of the arrow must be multiplied by the factor $\dot{q}|k|=$ $\ell d q / d t / u_{N}|k| \ll 1$, defined in [32], which measures the relative rate of change of the perturbation variable with respect to base-flow changes. It is observed that both fast-reaction and slow-reaction limits predict nearly similar qualitative results for the effect of exothermicity on the turbulent perturbations. It is only for sufficiently low Mach numbers that longitudinal kinetic energy exhibits an opposite response.

Figure 8 displays shaded contours for $L_{r}$ and $T_{r}$ as a function of $q$ and $M_{0} / M_{\mathrm{CJ}}$. Isocontour curves exhibit a nearly vertical trajectory for $q>3$, revealing a very weak dependence of the turbulent kinetic energy with heat release for relatively large values of $q$. It should be noticed that $q \sim 3$ is a relatively 

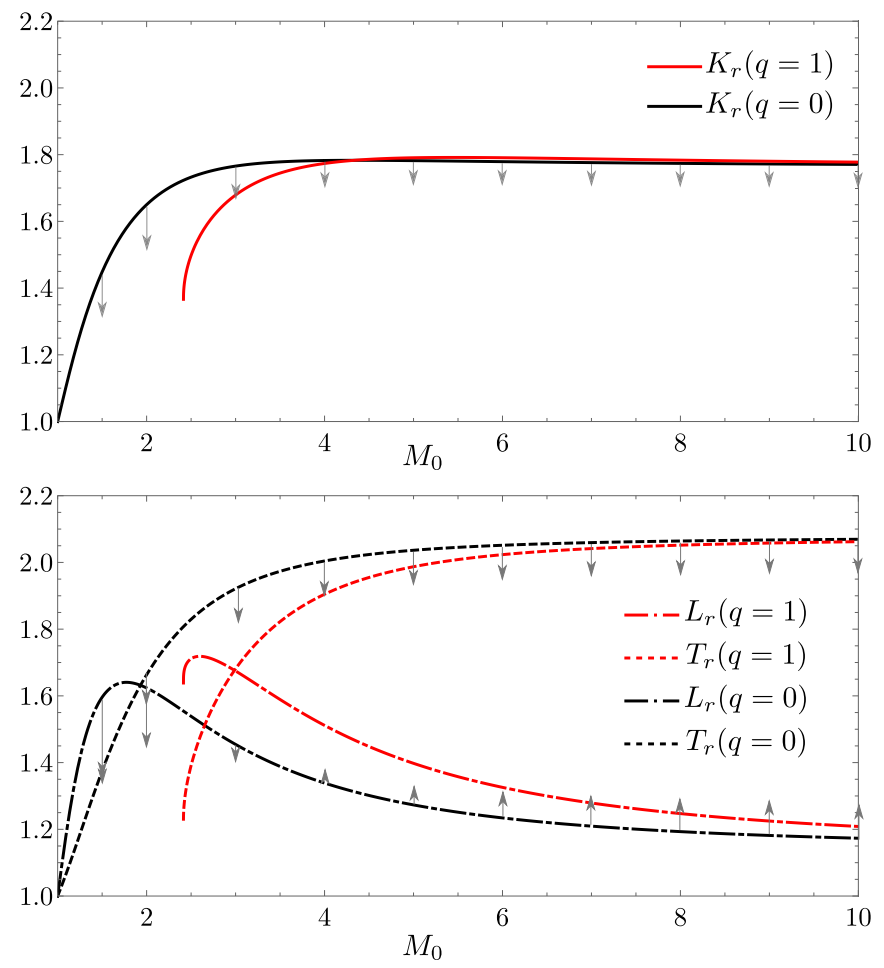

FIG. 7. $L_{r}$ (dotted-dashed line), $T_{r}$ (dashed line), and $K_{r}$ (solid line) as a function of the detonation Mach number $M_{0}$ for $\gamma=1.4$, $q=0$ (black), and $q=1$ (red). Arrows represent the effect of smallscale disturbances on the dominant nonreactive results [32].

small value in conventional reactive mixtures. As a matter of example, the dimensionless heat release of stoichiometric methane-air mixtures is about $q \sim 5$ in standard conditions. Both $L_{r}$ and $T_{r}$ approach a plateau regime for $M_{0} / M_{\mathrm{CJ}} \gg 1$, corresponding to the nonreactive strong-shock limit, whose analytical-closed expressions can be found in Ref. [18].

The linear fast-reaction theory is useful for predicting general trends and for quantifying scaling laws for the turbulent kinetic energy within a wide range of parameters. The breakdown of perturbations into the so-called Kovásznay modes enables the study of the kinetic energy, split into rotational and acoustic contributions, and the derivation of analytical-integral expressions. However, the model presented cannot address situations where the upstream characteristic length is of the same order as the detonation thickness and/or in where nonlinear changes arise. It is therefore convenient
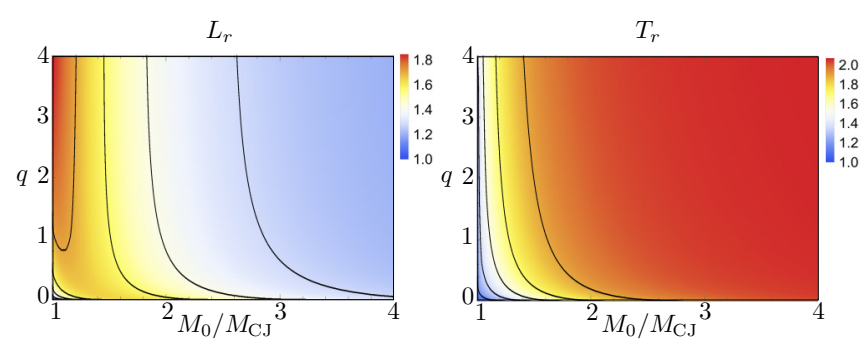

FIG. 8. Shaded contours for the longitudinal (left panel) and transverse (right panel) kinetic energy ratios as a function of $q$ and $M_{0} / M_{\mathrm{CJ}}$.
TABLE I. Computational parameters employed on the different numerical simulations performed.

\begin{tabular}{lccccccccc}
\hline \hline Case & $M_{t}$ & $q$ & $M_{0}$ & $\frac{M_{0}}{M_{\mathrm{CJ}}}$ & $\frac{E_{0}}{R_{g} T_{0}}$ & $\beta$ & $B(1 / \mathrm{ns})$ & $\ell(\mathrm{mm})$ & $\ell / \lambda_{t}$ \\
\hline NR & 0.1 & 0 & 3.62 & & & & & & \\
RU & 0.1 & 1 & 3.62 & 1.5 & 50 & 14.35 & 5519.1 & 0.047 & $\sim 1$ \\
RS1 & 0.1 & 1 & 3.62 & 1.5 & 10 & 2.87 & 0.0973 & 0.048 & $\sim 1$ \\
RS2 & 0.1 & 1 & 3.62 & 1.5 & 10 & 2.87 & 0.973 & 0.005 & $\sim 0.1$ \\
\hline \hline
\end{tabular}

to complement previous results with numerical simulations capable of capturing these effects. With this aim, the reactive Navier-Stokes equations are integrated in four distinguished scenarios that include the interaction of isotropic turbulence with a nonreactive shock (NR), a reactive unstable detonation (RU), reactive stable thick detonation (RS1), and a reactive stable thin detonation (RS2). The details of the numerical code and computations employed here can be found in Appendix B, with the computational parameters collected in Table I.

Numerical results of longitudinal and transverse components of the kinetic energy are shown in Fig. 9 as a function of the dimensionless streamwise coordinate $x_{t}$. The curves correspond to average-computed values along the two other orthogonal coordinates $y_{t}$ and $z_{t}$. The spatial coordinates are scaled with the Taylor microscale $\lambda_{t}$. The asymptotic value for the longitudinal and transverse kinetic energy amplification predicted by LIA is also shown. As expected, the amplitude of the perturbations in the reactive-unstable case is much stronger than those in stable situations. For stable situations, which are separately shown in the right-hand side panel of Fig. 9, the effect of heat release is found to increase the amplitude of perturbation right behind the shock front, with the smaller $\ell / \lambda_{t}$ exhibiting higher peaks. This effect is, however, diminished far behind the shock, product of the flow expansion and the strong
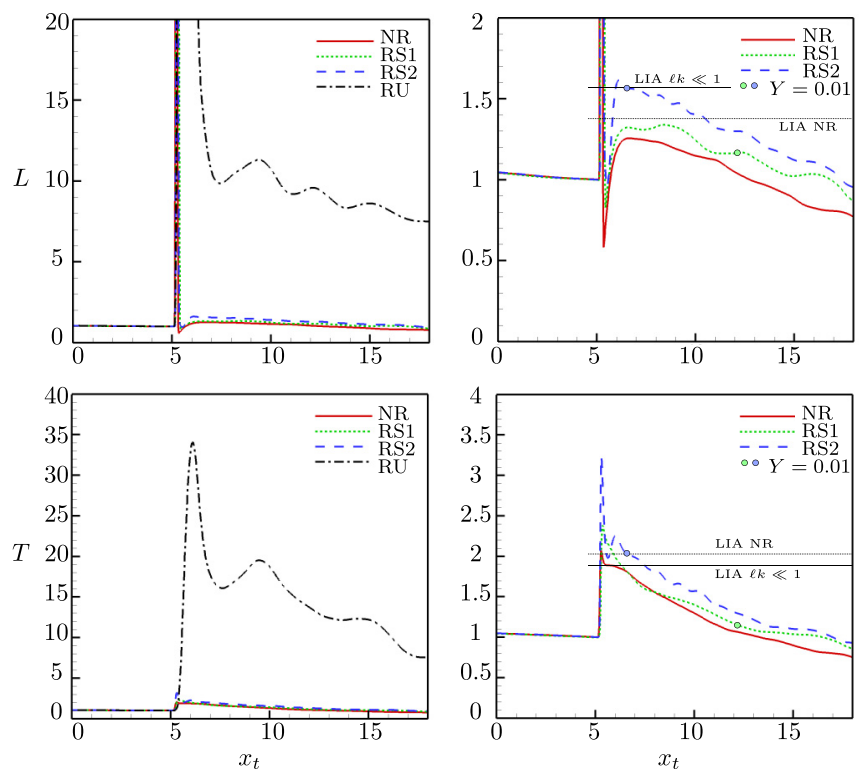

FIG. 9. DNS for the longitudinal (upper panels) and transverse (lower panels) kinetic energy, scaled with preshock values, as a function of the streamwise direction for $M_{0}=1.5 M_{\mathrm{CJ}}, q=1$, and $\gamma=1.4$. Circles denote nearly complete depletion of reactant $Y=$ 0.01. Left panels display the reactive unstable scenario RU. 

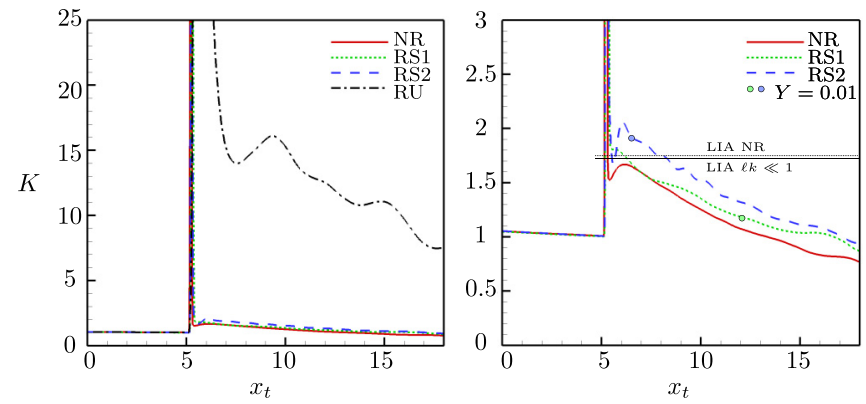

FIG. 10. DNS for the turbulent kinetic energy $K$, scaled with preshock values, as a function of the streamwise direction for $M_{0}=1.5 M_{\mathrm{CJ}}, q=1$, and $\gamma=1.4$. Circles denote nearly complete depletion of reactant $Y=0.01$. The left panel displays the reactive unstable scenario RU.

dissipative effects. The longitudinal and transverse kinetic energy shown in case RS1 is in qualitative agreement with LIA in the slow-reaction limit, where amplification factors at the end of the reactive zone are smaller than those generated by the shock front for the same Mach number $M_{0}$. Nonetheless, such comparison may be coincidental due to the strong numerical dissipation within the reaction zone. For case RS2, longitudinal kinetic energy amplification is found to be in fair agreement with LIA in the fast reaction limit, while crosswise kinetic energy computed by DNS is higher than that predicted by LIA.

The combination of longitudinal and transverse kinetic energy provides the total kinetic energy $K=(L+2 T) / 3$, shown in Fig. 10 for the same DNS cases used in Fig. 9. Since longitudinal and transverse kinetic energy display an opposite response to heat release in LIA, total kinetic energy shows little variation with heat release for this particular Mach number. The effect of detonation thickness in the burnt-gas turbulent kinetic energy is found to reduce the intensity of perturbations as a result of numerical dissipative effects. Right behind the shock, the turbulent kinetic energy is seen to be greater for shorter detonation thicknesses. When compared to nonreactive shocks, cases RS1 and RS2 predict higher turbulent energies for the same $x_{t}$, although case RS1 prognosticates smaller values for $K$ when looking at the values at the end of the reaction zone. As expected, reactive unstable detonations exceed the linear perturbation regime by one order of magnitude.

\section{B. Vorticity amplification factor}

The average rotational motion behind the detonation is appropriately measured by the so-called enstrophy $W$ defined as the square of the vorticity modulus, nondimensionalized with $\left(k a_{d}\right)^{2}$. As the normal component of the vorticity remains invariable after detonation passage, the total contribution is conveniently split into the longitudinal and perpendicular parts, namely,

$$
\begin{aligned}
W & =\frac{\left\langle\bar{\omega}_{x}^{2}+\bar{\omega}_{y}^{2}+\bar{\omega}_{z}^{2}\right\rangle}{\left\langle\bar{\omega}_{o, x}^{2}+\bar{\omega}_{o, y}^{2}+\bar{\omega}_{o, z}^{2}\right\rangle} \\
& =\frac{1}{3}+\frac{2}{3} \frac{\left\langle\bar{\omega}_{y}^{2}+\bar{\omega}_{z}^{2}\right\rangle}{\left\langle\bar{\omega}_{o, y}^{2}+\bar{\omega}_{o, z}^{2}\right\rangle}=\frac{1}{3}+\frac{2}{3} W_{\perp},
\end{aligned}
$$

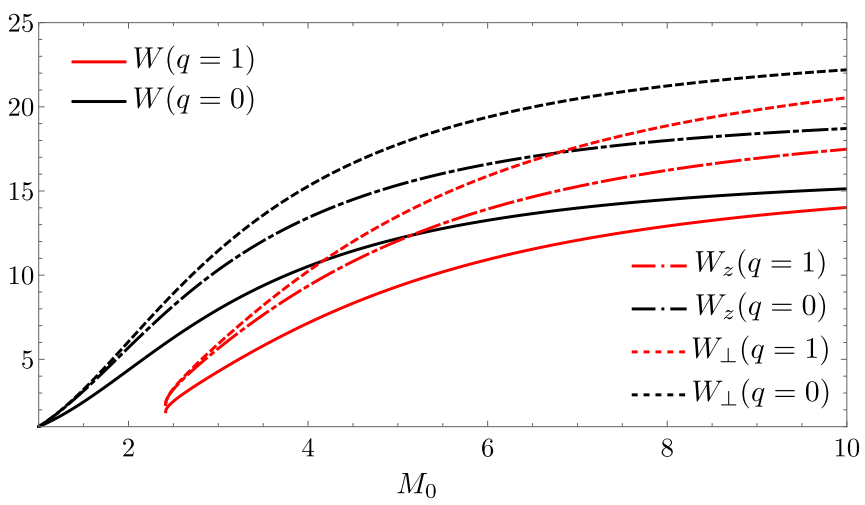

FIG. 11. $W_{z}$ (dotted-dashed line), $W_{\perp}$ (dashed line), and $W$ (solid line) as a function of the detonation Mach number $M_{0}$ for $\gamma=1.4$, $q=0$ (black), and $q=1$ (red).

with $W_{\perp}$ being the amplification factor of the averaged squared vorticity, normal to the direction of detonation motion. The two-dimensional equivalent factor

$$
W_{z}=\frac{\left\langle\bar{\omega}_{z}^{2}\right\rangle}{\left\langle\bar{\omega}_{o, z}^{2}\right\rangle}=\int_{0}^{\infty} \Omega^{2} \frac{R_{d}^{2} M_{d}^{2}}{R_{d}^{2} M_{d}^{2}+\left(1-M_{d}^{2}\right) \zeta^{2}} \mathrm{P}(\zeta) d \zeta
$$

is conveniently employed in computing $W_{\perp}=\left(R_{d}+3 W_{z}\right) / 4$. Computations for the breakdown components $W_{z}, W_{\perp}$, and $W$ are provided in Fig. 11 as a function of the detonation Mach number $M_{0}>M_{\mathrm{CJ}}$ for $\gamma=1.4, q=0$ (black), and $q=$ 1 (red). The curve for $W_{\perp}$ (black) is the similar to Fig. 5 in Ref. [14] for nonreacting shock waves in air $\gamma=1.4$. As $W_{z}$ and $R_{d}$ decreases when increasing $q$, the effect of heat release is found to be same for all contributions including $W$. This trend is in agreement with Fig. 4, where monochromatic vorticity perturbations are found to reduce their intensity with $q$ in the whole domain of the incident angle $\theta$. The lowering effect of exothermicity on downstream vorticity disturbances in the fast-reaction limit is found to be in qualitative agreement with slow-reaction-limit results [32].

Results of DNS are displayed in Fig. 12 for the same four scenarios investigated in Figs. 9 and 10. It is found that DNS for nonreactive (NR) and reactive-thin detonation (RS2) provide the following amplification factors for the perpendicular contribution: $W_{\perp} \sim 13.6$ and $W_{\perp} \sim 8$, respectively, which agree fairly well with LIA results 14.02 and 8.72 , respectively. The total vorticity amplification is also well captured by the numerical simulations, providing $W \sim 9.2$ and $W \sim 5.8$ for NR and RS2, which do not differ much from the values 9.7 and 6.1 obtained with the fast-reaction LIA. The case RS1, corresponding to relatively thick detonations, is affected by viscous dissipation within the reaction region, so that the level of enstrophy is relatively low when the combustion is completed. Excluding the unstable case, which is particularly different from the other scenarios, the values of the vorticity perturbations collapse far behind the leading shock due to viscous dissipation processes.

Further information is nonetheless depicted in the threedimensional (3D) computational results presented here. For instance, the mean vorticity perturbations $\omega_{t}=\left(\omega_{x}^{2}+\omega_{y}^{2}+\right.$ $\left.\omega_{z}^{2}\right)^{1 / 2}$, scaled with $u_{t} / \lambda_{t}$, are shown in Fig. 13 in a slice plane at the center of the computational box $y_{t}=(6.61 \pi) / 2$ 

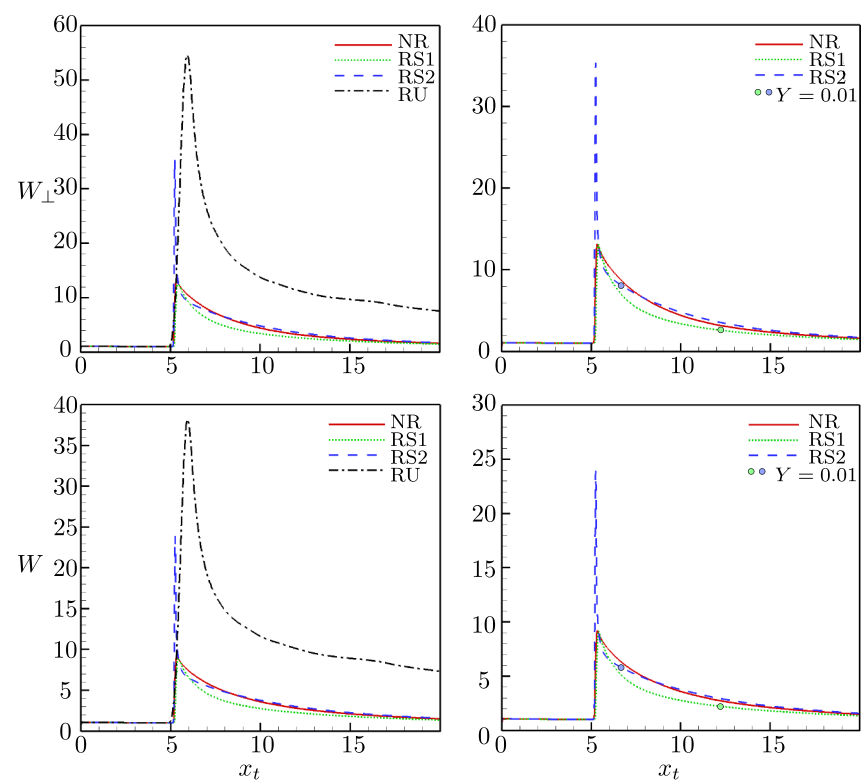

FIG. 12. DNS for the perpendicular (upper panels) and total (lower panels) enstrophy, scaled with preshock values, as a function of the streamwise direction for $M_{0}=1.5 M_{\mathrm{CJ}}, q=1$, and $\gamma=1.4$. Circles denote nearly complete depletion of reactant $Y=0.01$. Left panels display the reactive unstable scenario RU.

as a function of the streamwise $x_{t}=x / \lambda_{t}$ and vertical $z_{t}=$ $z / \lambda_{t}$ coordinates at a given instant. We can infer from the DNS output that both postshock (NR) and post-detonation (RU, RS1, RS2) vortical structures look qualitatively similar in all cases when excited with the same isotropic turbulent preshock flow (note the invariant field in $x_{t} \in[0,5]$ ), although they reveal several particular features of each computed case.
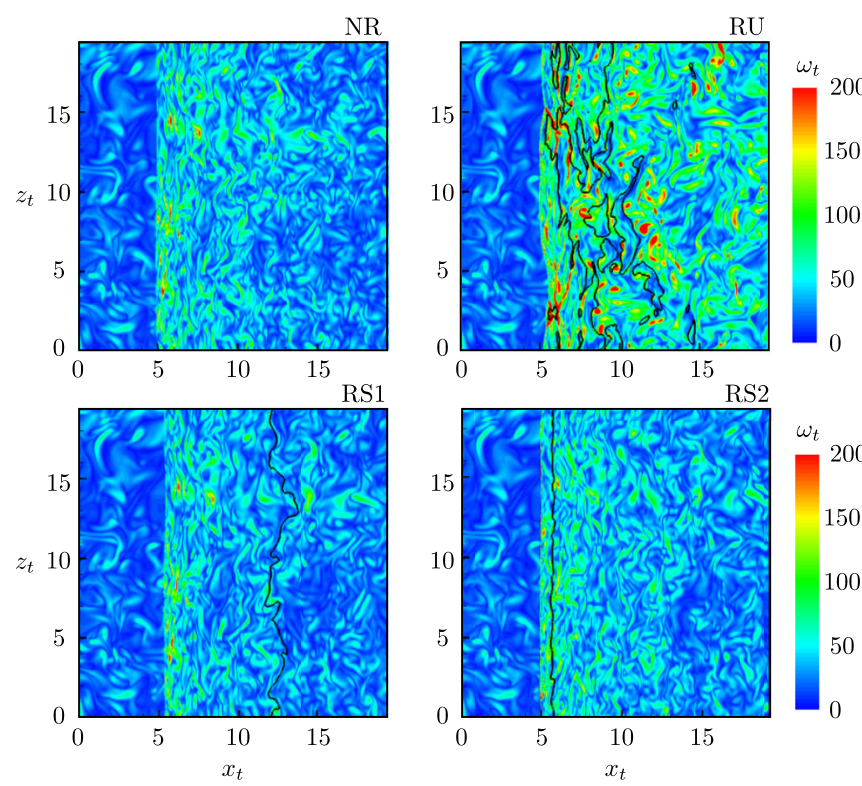

FIG. 13. Instantaneous vorticity patterns for NR, RU, RS1, and RS2 obtained at the cut plane $y_{t}=(6.61 \pi) / 2$. Black contour lines on the reactive cases correspond to the isolines of nearly complete depletion of reactant $Y=0.01$.
The energy liberated by reactant consumption conforms a thermal source that modifies the density of the fluid, advection capacity, and momentum transport coefficients, which come into an interplay with pressure perturbations to affect vortex structures. However, sufficiently weak reactions may not affect the vorticity field behind the shock wave to a substantial extent, as can be noted from comparison between NR and RS1-RS2 cases in Fig. 13, where structure size and composition are comparable as well as their amplitude. There, vortices are compressed across the shock wave and then slowly expanded due to the decay of turbulence. Nevertheless, sharper kinetics of the chemical reaction, given by drastic changes of the chemical-reaction parameters in RU, drive a stronger amplification of the vorticity field in addition to a higher spatial perturbation frequency in the transverse direction.

A measurement of combustion progress can be inferred from the mass fraction of reactant at each stage, essential to describe the detonation process. Isocontour lines are drawn on Fig. 13 to represent a $Y=0.01$ mass fraction of reactant, proving the unstable reaction to produce intricate structures of reacting scalar shown by the closed contour lines. These pockets of unburnt fuel are found further downstream than an earlier depletion stage of $Y=0.01$ and caused by strongly enhanced perturbations, which portrait the coupling between exothermic reactions and vorticity perturbations mentioned above. On the other hand, stable configurations display continuous depletion of scalar yielding a smooth character in terms of reaction process and localized continuous isocontour lines, which as shown in Fig. 9 are related to postshock longitudinal and transverse perturbations of the kinetic energy much smaller than in the precedent case. The moderately larger value of the frequency factor $B$ in RS2 with respect to RS1 $\left(B_{\mathrm{RS} 2} \sim\right.$ $10 B_{\mathrm{RS} 1}$ ) increases the amplitude of vortical perturbations but keeps their structure almost unaltered. However, consistent with this variation, the fuel-depletion distance from the shock is reduced from $\sim 7$ to $\sim 0.8$ length units in the streamwise direction.

In absence of perturbations, the evolution of the fuel mass fraction as a function of the distance from the shock is trivially obtained through the 1D integration of (8) and (9). Upstream perturbations, however, impose nonhomogeneous conditions that distort the detonation front and affect the average burning rate. Computations for the interaction of the planar reacting shock, in the four scenarios displayed in Table I with the upstream turbulent flow described above, provide averaged values for the turbulent half-reaction length $\ell_{\text {turb }}$. An unexpected outcome is that the value of the half-reaction length in turbulent conditions is found to be larger than that computed in perturbation-free conditions, providing $\ell_{\text {turb }} / \ell=$ $1.28,1.35$, and 1.23 for RU, RS1, and RS2, respectively. For sufficiently weak perturbations behind the shock, LIA results in the slow-reaction limit may apply. By direct inspection of the temperature perturbations behind the shock, $\bar{T}_{s} / \bar{p}_{s}=$ $\gamma-\left(M_{0}^{2} M_{d}^{2}\right)^{-1}$, it is found that they are mainly negative for incident angles below $\theta_{c r}$ and positive otherwise. It gives the possibility of finding both accelerating or decelerating regimes in the overall burning rate depending on the upstream spectrum. In the high-activation-energy limit $\beta \gg 1$, where the reaction region is clearly differentiated from the induction zone, the amplitude of the weak perturbations $\epsilon$ might be 
of the same order as $\beta^{-1} \ll 1$, then leading to order-unity changes in the reaction rate. Consequently, the reaction front and the leading shock are effectively coupled by the entropic and acoustic modes. The Newtonian approach $\gamma-1 \ll 1$ provides two distinguished limits: strongly overdriven detonations (entropic coupling) or low-exothermic detonation in CJ conditions (acoustic coupling) [37]. The computations conducted in this work are placed within these two limits.

\section{Anisotropy degree}

The effect of the detonation passage on the turbulent kinetic energy, computed in Figs. 6-8, reveals different responses for longitudinal and transverse contributions, then breaking the predetonation isotropic condition. After detonation, it is found that the kinetic energy of the perturbations is mainly stored in transverse perturbations for sufficiently strong detonations. In order to compute the degree of anisotropy downstream, the factor

$$
\Psi=\frac{\left\langle\bar{v}_{\perp}^{2}\right\rangle-2\left\langle\bar{v}_{\|}^{2}\right\rangle}{\left\langle\bar{v}_{\perp}^{2}\right\rangle+2\left\langle\bar{v}_{\|}^{2}\right\rangle}=1-\frac{4\left\langle\bar{u}^{2}\right\rangle}{\left\langle\bar{v}^{2}\right\rangle+\left\langle\bar{u}^{2}\right\rangle}=1-\frac{4 L}{3 K+L}
$$

is conveniently introduced in a similar fashion as in Ref. [18]. This factor is bounded by $\Psi=-1$ and +1 , with the former representing turbulent flows dominated by streamwise velocity perturbations. The opposite limit represents turbulent flows whose longitudinal contribution is negligible. The value $\Psi=0$ then represents isotropic velocity perturbations downstream.

The anisotropy factor is quantified in Fig. 14 for nonreactive and reactive shock waves as a function of $M_{0} / M_{\mathrm{CJ}}-1$. In order to characterize the degree of anisotropy corresponding to rotational disturbances only, the value of $\Psi_{r}=$ $1-4 L_{r} /\left(3 K_{r}+L_{r}\right)$ is also computed. The graph reveals that lateral contribution is dominant for most representative situations, being the longitudinal contribution dominant for weak detonation conditions only, where acoustic influences peak, in consonance with Fig. 6. A distinguishing feature of the reacting case is that $\Psi$ is a monotonic function of $M_{0} / M_{\mathrm{CJ}}$, departing from a longitudinal-dominant turbulent flow. Computations for the effect of the adiabatic index show

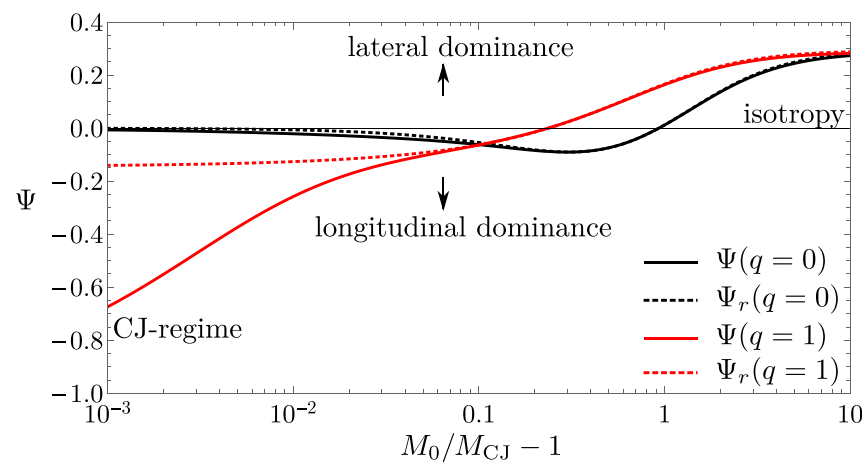

FIG. 14. Anisotropy factor $\Psi$ as a function of the overdrive $M_{0} / M_{\mathrm{CJ}}-1$ for nonreactive (black) and reactive (red) cases. Dotted lines represent the value of the rotational anisotropy factor $\Psi_{r}$. that low-compressible gases $\gamma \sim 2$ are found to remain mainly isotropic after shock (detonation) passage for finite shock (detonation) intensities. It is worth commenting that $\Psi=1$ is theoretically obtained in the high-compressibility strongwave limit $\gamma-1 \ll 1$ and $M_{0} \gg 1$. Isotropic conditions downstream are marginally found; for instance, LIA predicts $\Psi=0$ at $M_{0}=1.93$ for nonreacting shock waves, while reacting shock waves with $q=1$ are found to create isotropic turbulent flows for $M_{0} / M_{\mathrm{CJ}}=1.24\left(M_{0}=2.98\right)$. Numerical simulations show that dissipative effects, when considered, do not play any significant role in the energy transfer between streamwise and lateral perturbations, then rendering a degree of anisotropy that remains fairly constant with time $[17,22]$.

\section{General comments on LIA and DNS results}

Quantitative comparison between DNS and LIA results is a difficult task even for canonical configurations. LIA does not account for nonlinear nor viscous effects and fails in reproducing the wide range of scales involved if a reactive flow is considered. On the other hand, DNS account for viscous dissipation, which makes the turbulent kinetic energy decay behind the leading shock. DNS also encounter difficulties in reproducing ideal discontinuities. The former issue was addressed in Larsson et al. [22], who proposed a modified far-field turbulent kinetic energy computed by neglecting the dissipation term, which renders results in fairly good agreement with LIA. The latter aspect was investigated in Ref. [23], and it was found that only when there is a significant difference in scale between the shock thickness and the incoming disturbances, viscous and nonlinear effects become negligible during the interaction process, making LIA coherent with DNS. This condition, which is not easily satisfied in computations due to numerical-diffusive effects reproducing the shock front, is even harder to fulfill when detonation waves are contemplated.

It is known that for nonreactive shock waves, LIA estimations, in comparison with DNS results, overpredict the intensity of lateral disturbances and underpredict the longitudinal perturbations, although total kinetic energy rendered is in good agreement $[17,22]$. Compared to previous results found in literature, however, the effect of heat release on the turbulent kinetic energy does not portray a clear trend. For instance, the thick-detonation limit developed in Ref. [32] and finitereaction-rate numerical results performed in Refs. [29,30] predict a lowering effect of exothermicity on the longitudinal kinetic energy for sufficiently low Mach numbers. These numerical investigations show that solutions with nonharmonic response may arise when the size of upstream perturbations are of the same order of the detonation thickness, for which the present fast-chemistry linear analysis would become clearly inaccurate. It suggests that perturbation dynamics within the reaction region is pivotal, resulting in lowering or increasing streamwise perturbations when the reaction occurs gradually or suddenly behind the shock, respectively. By way of contrast, both thin- and thick-detonation limits agree in the lowering effect of exothermicity in the transverse kinetic energy and in the amplitude of the average vorticity disturbances. When looking at the amplitude of the vorticity perturbations behind 
the detonation, both nonreactive and fast-reaction-limit results obtained through LIA agree fairly well with DNS. This accordance is better than that seen for the turbulent kinetic energy computations.

A plausible explanation for the almost-general lessening effect of heat release prognosticated by LIA might be rendered by the mass compression ratio factor $R_{d}$, which is always smaller than that found across the shock due the postshock gas expansion. This is clearly observed when looking at the post-detonation vorticity perturbations since a dominant contribution of the vortex transformation is due to the shrinking response across the detonation front. As shown in Figs. 4 and 5, weaker overdrives result in stronger longitudinal velocity perturbations, yet weaker transverse velocity and vorticity disturbances. In addition, the globally exothermic reaction involved in the combustion process is sensitive to temperature perturbations as those generated across the shock front. The leading shock, subsonic with respect to the reacting gas, responds to such perturbations, then conforming an acoustically coupled system that evolves with time as shown in Fig. 13. For relatively thin detonations $\ell / \lambda_{t} \sim 0.1$, a value that still stands far from being the idealized discontinuity condition [23], the amplitude of turbulent perturbations is found to be higher than that computed when $\ell / \lambda_{t} \sim 1$, and then closer to those values predicted by LIA as shown for the longitudinal kinetic energy on Fig. 9. Further studies, as those conducted in [23], would be needed in this direction to confirm that LIA results are reliable in a wider range of parameters.

\section{CONCLUSIONS}

The LIA on detonation-turbulence interaction is an extension to previous theoretical works $[27,28,31]$ that includes transient histories for single-mode perturbations and overall transfer functions associated with isotropic vorticity spectra. The results presented, including explicit analytic formulas for all quantities of interest, are used to test the linear response of detonations to weak vorticity perturbations, for conditions that render the basic planar solution stable with the characteristic size of the disturbances much larger than the detonation thickness. The effect of overdrive and relative heat release on the turbulent kinetic energy, vorticity, and anisotropy degree has been investigated in a wide range of the parametric domain. It is found that the heat release in the detonation mostly reduces the relative fluctuation intensities downstream below the levels that would exist behind the inert leading shock, in consonance with the thick-detonation limit [32]. In terms of the qualitative response of the detonation against weak perturbations, it is found that downstream disturbances are mainly generated through the leading wrinkled shock for small-scale perturbations [32], and across the detonation front for large-scale perturbations.

DNS are performed to take into consideration the effect of similar-size perturbations and nonstable dynamics in the detonation evolution. These two effects are incorporated in the model through the frequency-collision factor and the activation energy in the reaction rate equation. Numerical viscous dissipation within the reaction zone hampers the comparison with LIA. An expected finding is that nonstable detonation waves, those occurring in detonations characterized by weak overdrive factors $M_{0} / M_{\mathrm{CJ}}-1 \ll 1$ and relatively high activation energy $E_{a} /\left(R_{g} T_{0}\right) \gg 1$, generate downstream perturbations that are stronger, by one order of magnitude, than upstream perturbations. They are not a result of upstream excitement but rather of self-induced oscillations that ultimately lead to very strong waves traveling perpendicular to the leading shock. By way of contrast, when the detonation is stable (steady for perturbation-free conditions), the amplitude of the perturbations generated through the detonation wave is of the same order as upstream perturbations. For the cases considered in the computations, heat release is found to increase the amplitude of the perturbations right behind the shock front, with respect to the nonreactive case with similar propagation Mach number. The effect of detonation thickness reveals that thicker detonations generate weaker turbulent fluctuations right behind the shock.

\section{ACKNOWLEDGMENTS}

This work was supported by the Ministry of Science, MEC (Grant No. ENE2015-65852-C2-1-R), and Fundación Iberdrola España (BINV-ua37crdy), Spain (C.H. and D.M-R), by the National Natural Science Foundation of China (Grants No. 51576176 and No. 91541202) and by the Special Program for Applied Research on Super Computation of the NSFCGuangdong Joint Fund (T.J. and K.L).

\section{APPENDIX A: TRANSIENT EVOLUTION FOR TWO-DIMENSIONAL MONOCHROMATIC VORTICAL DISTURBANCES}

Computations for the interaction with 3D isotropic turbulent flows consider the long-time response of the detonation wave as that responsible of the perturbations downstream. It is therefore convenient to analyze the transient response of the detonation wave so that the validity of asymptotic analysis is quantified.

Figure 15 depicts the interaction of the detonation front with the shear-wave sheet described in (11). Once the detonation enters the perturbation region it is distorted and a set of acoustic, entropic, and vortical perturbations are generated behind the front. To describe burnt-gas flow dynamics, the temporal evolution of the detonation front must be resolved

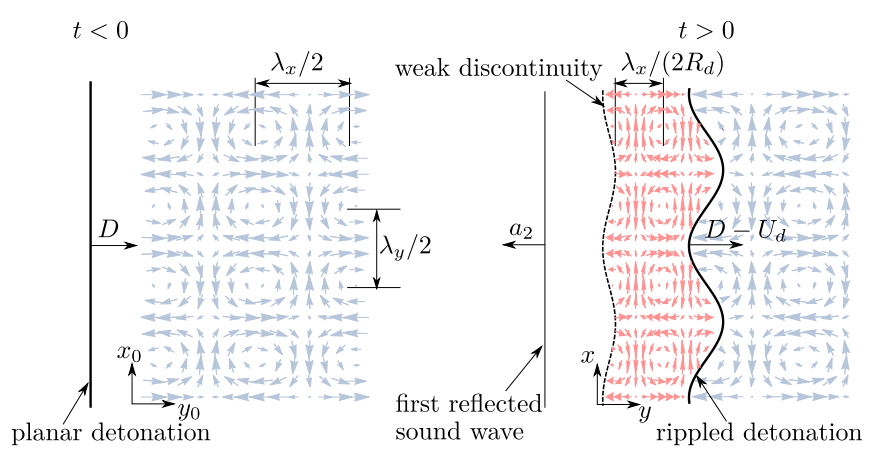

FIG. 15. Sketch of the interaction of an initially planar detonation wave with a monochromatic vorticity field. 
from $\tau \geqslant 0$, with $\tau=0$ being the moment at which the initially planar detonation front encounters the perturbed field. This evolution is governed by the sound wave equation (15) that couples the detonation front with the burnt-gas conditions. To this end, a similar approach as that used in Ref. [18] is employed here, with the hyperbolic transformation

$$
k_{y} x=r \sinh \chi, \quad \tau=r \cosh \chi
$$

conveniently applied. It is readily seen that $\chi=$ constant represents a planar front moving in the burnt gas along the $x$ axis, then sweeping the burnt-gas domain from the weak discontinuity $k_{y} x=0(\chi=0)$ to the detonation front $k_{y} x=M_{d} \tau\left(\tanh \chi_{d}=M_{d}\right)$. The sound-wave equation (15) reduces to

$$
r \frac{\partial^{2} \bar{p}}{\partial r^{2}}+\frac{\partial \bar{p}}{\partial r}+r \bar{p}=\frac{1}{r} \frac{\partial^{2} \bar{p}}{\partial \chi^{2}}
$$

while the detonation boundary condition at the front becomes

$$
\frac{d \bar{\xi}}{d r}=\frac{R_{d}\left(1-\Pi_{d}\right)}{2\left(R_{d}-1\right) M_{d} \sqrt{1-M_{d}^{2}}} \tilde{p}_{d}+\frac{1}{\sqrt{1-M_{d}^{2}}} \cos (\zeta r),
$$

$$
\begin{aligned}
\frac{1}{r} \frac{\partial \bar{p}}{\partial \chi}= & -\frac{1+\Pi_{d}}{2 M_{d}} \frac{\partial \bar{p}}{\partial r}-\frac{M_{d}^{2}\left(R_{d}-1\right)}{\sqrt{1-M_{d}^{2}}} \bar{\xi} \\
& +\frac{\left(R_{d}-1\right) \zeta}{R_{d}} \sin (\zeta r) .
\end{aligned}
$$

The development continues by applying the Laplace transform to (A2) and (A3). In particular, as shown previously [18], the condition that the detonation remains isolated from downstream influences can be used in (A3) to generate from (A2) the algebraic equations

$$
\begin{gathered}
s \Xi_{d}(s)=\frac{R_{d}\left(1-\Pi_{d}\right)}{2\left(R_{d}-1\right) M_{d} \sqrt{1-M_{d}^{2}}} P_{d}(s)-\frac{1}{\sqrt{1-M_{d}^{2}}} \frac{s}{\zeta^{2}+s^{2}}, \\
\sqrt{s^{2}+1} P_{d}(s)-\tilde{p}_{d_{i}}=-\frac{1+\Pi_{d}}{2 M_{d}}\left[s P_{d}(s)-\tilde{p}_{d_{i}}\right]-\frac{M_{d}^{2}\left(R_{d}-1\right)}{\sqrt{1-M_{d}^{2}}} \Xi_{d}(s)+\frac{\left(R_{d}-1\right) \zeta}{R_{d}} \frac{\zeta^{2}}{\zeta^{2}+s^{2}},
\end{gathered}
$$

where

$$
P_{d}(s)=\int_{0}^{\infty} \bar{p}_{d}(r) \exp (-s r) d r, \quad \Xi_{d}(s)=\int_{0}^{\infty} \bar{\xi}_{d}(r) \exp (-s r) d r
$$

are the Laplace transforms of $\tilde{p}_{d}$ and $\bar{\xi}$ with respect to the variable $r$, respectively, and $\tilde{p}_{d_{i}}$ is the initial pressure perturbation at the detonation front.

Eliminating $\Xi_{d}(s)$ and solving for $P_{d}(s)$ yields

$$
\begin{aligned}
P_{d}(s)= & \frac{\left(1-M_{d}^{2}\right)\left(2 M_{d}+1+\Pi_{d}\right) s}{2 M_{d}\left(1-M_{d}^{2}\right) s \sqrt{s^{2}+1}+\left(1+\Pi_{d}\right)\left(1-M_{d}^{2}\right) s^{2}+\left(1-\Pi_{d}\right) R_{d} M_{d}^{2}} \tilde{p}_{d_{i}} \\
& +\frac{2 M_{d}\left(1-M_{d}^{2}\right)\left(1-R_{d}^{-1}\right)}{2 M_{d}\left(1-M_{d}^{2}\right) s \sqrt{s^{2}+1}+\left(1+\Pi_{d}\right)\left(1-M_{d}^{2}\right) s^{2}+\left(1-\Pi_{d}\right) R_{d} M_{d}^{2}}\left(\zeta^{2}-\frac{R_{d} M_{d}^{2}}{1-M_{d}^{2}}\right) \frac{s}{\left(s^{2}+\zeta^{2}\right)}
\end{aligned}
$$

The transient evolution for the pressure perturbation behind the detonation wave is determined by the inverse Laplace transform of (A6), and it is found that

$$
\bar{p}_{d}(\tau)=-\frac{2}{\pi} \int_{0}^{1} \cos \left[z\left(1-M_{d}^{2}\right)^{1 / 2} \tau\right] f(z) d z+\frac{2\left(R_{d}-1\right)}{\pi R_{d}}\left(\zeta^{2}-\frac{M_{d}^{2} R_{d}}{1-M_{d}^{2}}\right) \int_{0}^{1} \frac{\cos \left[z\left(1-M_{d}^{2}\right)^{1 / 2} \tau\right]-\cos \left(\omega_{d} \tau\right)}{\zeta^{2}-z^{2}} f(z) d z
$$

with

$$
f(z)=\frac{4 M_{d}^{2}\left(1-M_{d}^{2}\right)^{2} z \sqrt{1-z^{2}}}{4 M_{d}^{2}\left(1-M_{d}^{2}\right)^{2} z^{2}\left(1-z^{2}\right)^{2}+\left[\left(1-M_{d}^{2}\right)\left(1+\Pi_{d}\right) z^{2}-M_{d}^{2} R_{d}\left(1-\Pi_{d}\right)\right]^{2}}
$$

being an auxiliary integration function. The shock-oscillation frequency $\omega_{d}=\zeta\left(1-M_{d}^{2}\right)^{1 / 2}$. The solution given in (A7) describes the evolution of the detonation pressure for $\tau>0$, which approaches for $\tau \gg 1$ a periodic solution [see Eq. (19)], in which the detonation front oscillates harmonically with a frequency proportional to $\zeta$ with a constant amplitude.

It is worth pointing out that the rate at which the transient solutions (A7) approach the permanent periodic solutions for large times is determined by the branch point associated with $\sqrt{1-z^{2}}$ in (A8), i.e., with $\sqrt{s^{2}+1}$ in (A6), which represents the generation of evanescent sound-wave perturbations that in the general case decay asymptotically in time like $\tau^{-3 / 2}$. Observation of (A7) and (A8) indicates that this decay rate changes, however, for infinitely strong detonations with $M_{0} \rightarrow$ $\infty$, for which, from (A6), poles lay on the branch points since $R_{d} \rightarrow(\gamma+1) /(\gamma-1), M_{d} \rightarrow \sqrt{(\gamma-1) /(2 \gamma)}, \kappa \rightarrow 1$, 

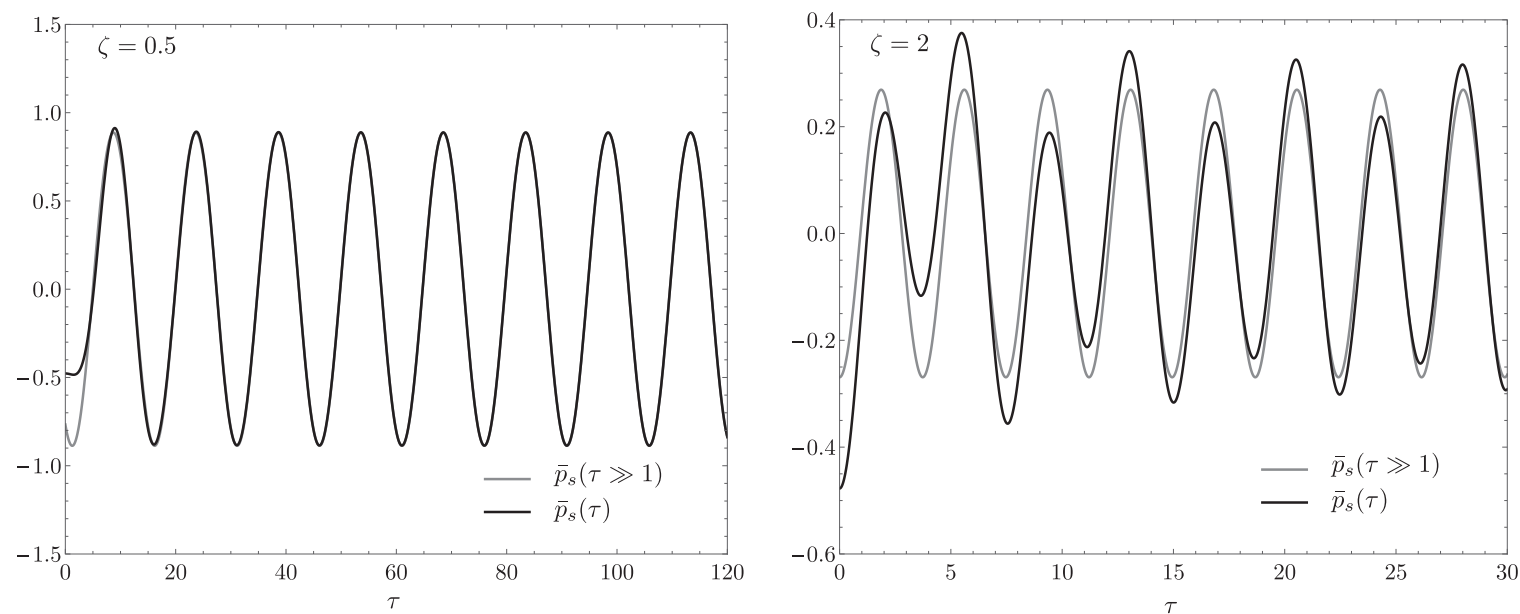

FIG. 16. Temporal evolution of pressure perturbations behind the detonation front traveling at $M_{0}=1.5 M_{\mathrm{CJ}}$, for $q=1$ and $\gamma=1.4$, for two different upstream frequencies $k_{x} / k_{y}$.

and $\Pi_{d} \rightarrow 0$. In this limit, the decay towards the permanent solution would follow a $\tau^{-1 / 2}$ law, as determined by the new mathematical structure of the integration function $f(z)$, which develops a factor $\sqrt{1-z^{2}}$ in the denominator. This effect was first noticed by Fraley [44] for very strong shock waves, and it is also recovered here for strong detonations. In addition, irrespective of the strength of the detonation, the modes with incident frequency $\zeta=1$ also show a slow decay from the initial solution, proportional to $\tau^{-1 / 2}$, as can be inferred from the factor $\zeta^{2}-z^{2}$ appearing in the denominator of the second integrals in (A7). Modes with values of $\zeta$ close to $\zeta=1$ will therefore decay more slowly than those with either higher or lower frequencies.

In Fig. 16 it is displayed the detonation pressure evolution as a function of $\tau$ predicted by linear theory in the fast-reaction limit for two different regimes: high-frequency $(\zeta>1)$ and low-frequency perturbations $(\zeta<1)$. Similarly as found in $[18,31]$, the decay of the transient evolution towards the asymptotic solution follows a $\tau^{-3 / 2}$ law for $\zeta \neq 1$. For $\zeta=1$, however, the decay follows a $\tau^{-1 / 2}$ law as determined by the new mathematical structure of the integration function (A8) in this limit, then suggesting that the peak shown in Figs. 2, 5, and 4 may not represent realistic conditions for the long-time response. This, however, has very little impact on turbulent kinetic energy averages.

The detonation rippling evolution is easily derived from the linearized RH equations, yielding

$$
\begin{aligned}
\bar{\xi}(\tau)= & -\frac{2}{\pi} \int_{0}^{1} \frac{\sin \left[z\left(1-M_{d}^{2}\right)^{1 / 2} \tau\right]}{z\left(1-M_{d}^{2}\right)^{1 / 2}} f(z) d z+\frac{\sin \left(\omega_{d} \tau\right)}{\omega_{d}} \\
& +\frac{2\left(R_{d}-1\right)}{\pi R_{d} \omega_{d}}\left(\zeta^{2}-\frac{M_{d}^{2} R_{d}}{1-M_{d}^{2}}\right) \\
& \times \int_{0}^{1} \frac{\zeta \sin \left[z\left(1-M_{d}^{2}\right)^{1 / 2} \tau\right]-z \sin \left(\omega_{d} \tau\right)}{z\left(\zeta^{2}-z^{2}\right)} f(z) d z
\end{aligned}
$$

The transient effects of the detonation front in the burnt-gas flow can be also addressed with analytical techniques. In particular, the pressure field is described, in terms of the Laplace variable $s$ as

$P_{d}(s, \chi)=\frac{\sqrt{s^{2}+1} \cosh \left(\chi_{d}-\chi\right)+s \sinh \left(\chi_{d}-\chi\right)}{\sqrt{s^{2}+1}} \hat{P}_{d}(s, \chi)$,

with $\hat{P}_{d}(s, \chi)$ being the Laplace transform of the pressure perturbations behind the detonation (A10), but evaluated at $P_{d}\left[s=s \cosh \left(\chi_{d}-\chi\right)+\sqrt{s^{2}+1} \sinh \left(\chi_{d}-\chi\right)\right]$. The pressure perturbation field in Cartesian coordinates $k_{y} x$ and $k_{y} y$ is obtained through the inverse Laplace transform of (A10) and using the hyperbolic transformation shown in (A1), namely, $r=\sqrt{\tau^{2}-\left(k_{y} x\right)^{2}}$ and $\chi=\tanh ^{-1}\left(k_{y} x / \tau\right)$. The distinguished nonradiating and radiating regimes are clearly identified in Fig. 17, where the pressure field has been computed for $\zeta<1$ (left) and $\zeta>1$ (right). For low-frequency oscillations, the amplitude of the pressure perturbations at the detonation front exponentially decays behind it. On the other hand, stable sonic radiation is obtained for high-frequency oscillations, as predicted by the asymptotic analysis described in (28) and the posterior paragraph. Although not shown in the paper, DNS have been performed with the same numerical parameters as in Fig. 17. Both nonradiating and radiating regimes are well captured by the simulations, with the only difference that far-field amplitudes decay due to numerical dissipative effects.

As it has been shown in [18], the vorticity generated behind the detonation wave at $k_{y} x=0$ seeds the unstable rippling of the weak surface separating two clearly distinguished regions: the vorticity-free zone within $-\tau<k_{y} x<0$ (as reflected sound waves that perturb this space are irrotational) and the rotational region within $0<k_{y} x<M_{d} \tau$ (see sketch in Fig. 15). The weak discontinuity evolves as a RichtmyerMeshkov unstable surface, with the amplitude of wrinkled surface growing linearly with time. From that point forth, the instantaneous vorticity and entropy disturbances deposited by the detonation wave within the space $0<k_{y} x<M_{d} \tau$ remain frozen to the fluid particles, in absence of diffusive effects. 

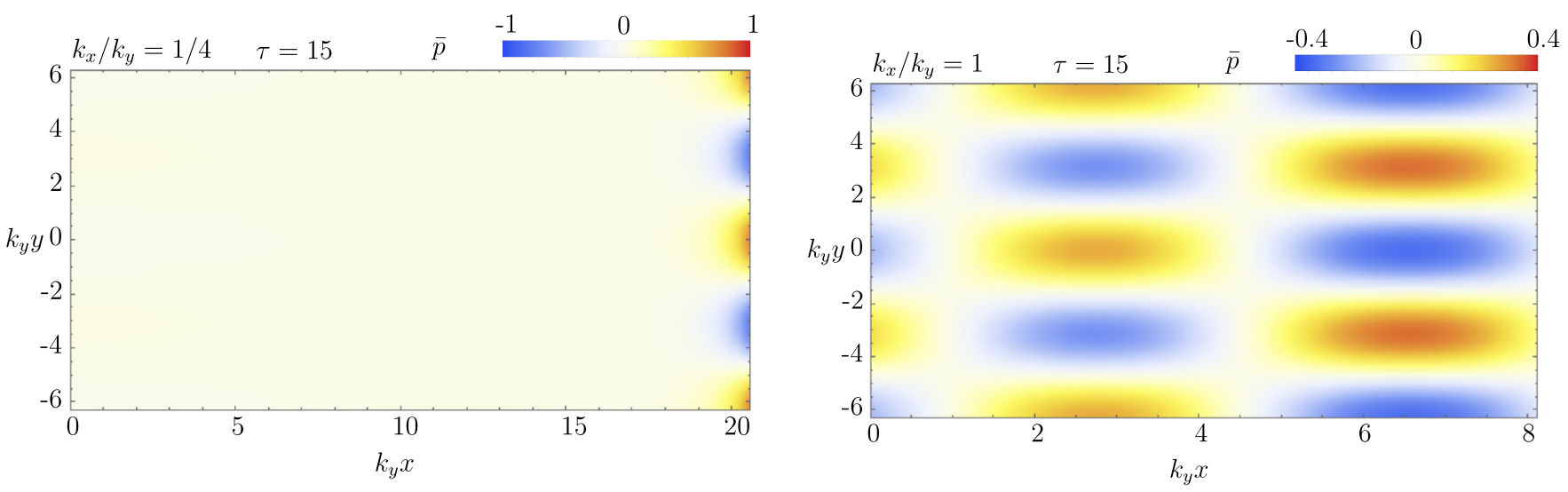

FIG. 17. Spatial distribution of pressure perturbations in the burnt gas for a detonation front traveling at $M_{0}=1.5 M_{\mathrm{CJ}}$, with $q=1$ and $\gamma=1.4$, and for two different upstream frequency ratios $k_{x} / k_{y}=1 / 4$ (left) and 1 (right).

The linear perturbations in the velocity field satisfy the equation

$$
\frac{\partial^{2} \overrightarrow{\vec{v}}}{\partial \tau^{2}}=\nabla \times \nabla \times \overrightarrow{\vec{v}}+\nabla^{2} \overrightarrow{\vec{v}}
$$

with the breakdown of irrotational-acoustic and steadyrotational perturbations obeying, separately,

$$
\frac{\partial^{2} \overrightarrow{\vec{v}}_{a}}{\partial \tau^{2}}=\nabla^{2} \overrightarrow{\bar{v}}_{a} \quad \text { and } \quad \nabla^{2} \overrightarrow{\bar{v}}_{r}=\frac{\partial \bar{\omega}}{\partial\left(k_{y} y\right)} \hat{e}_{x}-\frac{\partial \bar{\omega}}{\partial\left(k_{y} x\right)} \hat{e}_{y} .
$$

The function $\bar{\omega}(x, y)=g\left(k_{y} x\right) \sin \left(k_{y} y\right)$, with $g\left(k_{y} x\right)$ provided in (36), represents the spatial distribution of the vorticity field. It is seen that rotational perturbations are incompressible, and then isobaric in the linear limit. The acoustic perturbations are readily obtained through the Euler momentum equations

$$
\frac{\partial \bar{u}_{a}}{\partial \tau}=-\frac{\partial \bar{p}}{\partial\left(k_{y} x\right)} \quad \text { and } \quad \frac{\partial \bar{v}_{a}}{\partial \tau}=\bar{p},
$$

with the pressure function $\bar{p}$ being computed in Fig. 17. The spatial distribution of the rotational-velocity perturbations is calculated by tracking the vorticity left behind by the oscillating detonation front from $\tau=0$. As described in Ref. [18], the solenoidal disturbances generated by the detonation wave are

$$
\begin{gathered}
\bar{u}_{r}(x, y)=\left[\bar{u}_{p}+\frac{\exp \left(-k_{y} x\right)}{\sqrt{1-M_{d}^{2}}} P_{d}\left(s=\sinh \chi_{d}\right)\right] \cos \left(k_{y} y\right), \\
\bar{v}_{r}(x, y)=\left[\frac{\partial \bar{u}_{p}}{\partial\left(k_{y} x\right)}-\frac{\exp \left(-k_{y} x\right)}{\sqrt{1-M_{d}^{2}}} P_{d}\left(s=\sinh \chi_{d}\right)\right] \sin \left(k_{y} y\right),
\end{gathered}
$$

with the particular solution $\bar{u}_{p}$ being provided by

$$
\begin{aligned}
\bar{u}_{p}\left(k_{y} x\right)= & -\frac{2 \Omega_{2}}{\pi} \int_{0}^{1} f(z) \frac{\cos \left[\left(M_{d}^{-2}-1\right)^{1 / 2} z k_{y} x\right]}{1+\left(M_{d}^{-2}-1\right) z^{2}} d z \\
& +\frac{2 \Omega_{2}\left(1-R_{d}^{-1}\right)}{\pi}\left(\zeta^{2}-\frac{R_{d} M_{d}^{2}}{1-M_{d}^{2}}\right)
\end{aligned}
$$

$$
\begin{aligned}
& \times \int_{0}^{1} \frac{f(z)}{\zeta^{2}-z^{2}}\left\{\frac{\cos \left[\left(M_{d}^{-2}-1\right)^{1 / 2} z k_{y} x\right]}{1+\left(M_{d}^{-2}-1\right) z^{2}}\right. \\
& \left.-\frac{\cos \left[\left(M_{d}^{-2}-1\right)^{1 / 2} \zeta k_{y} x\right]}{1+\left(M_{d}^{-2}-1\right) \zeta^{2}}\right\} d z \\
& +\Omega_{1} \frac{\cos \left[\left(M_{d}^{-2}-1\right)^{1 / 2} \zeta k_{y} x\right]}{1+\left(M_{d}^{-2}-1\right) \zeta^{2}} .
\end{aligned}
$$

Similarly, provided that $\bar{p}_{d}(\tau)$ is given in (A7), the entropic perturbations generated by the detonation wave are

$$
\bar{\rho}_{e}\left(k_{y} x\right)=\frac{\Pi_{d}-M_{d}^{2}}{M_{d}^{2}} \bar{p}_{d}\left[\tau=\left(M_{d}^{-2}-1\right)^{1 / 2} k_{y} x\right],
$$

with $\bar{p}_{d}$ obtained previously in (A7). It is easily seen that $\bar{\rho}_{e}=0$ in absence of pressure perturbations, i.e., in neutral transmission conditions occurring for $\zeta=\zeta^{*}$.

The upper panel in Fig. 18 displays the transient and asymptotic predictions for rotational and entropic disturbances as a function of the streamwise coordinate $k_{y} x$. The conditions are $M_{0}=1.5 M_{\mathrm{CJ}}, M_{0}=1.5 M_{\mathrm{CJ}}, q=1, \gamma=1.4$, and $\zeta=1.5$. It is observed that the amplitude of $\bar{\rho}_{e}$ decays asymptotically towards $\mathcal{P}\left(\Pi_{d}-M_{d}^{2}\right) / M_{d}^{2}=0.074$. The rotational velocity field is found to match the asymptotic solution in a shorter distance. The lower panel shows the two-dimensional vector field plot for rotational-velocity perturbations superposed to the isocontours of the entropic-density disturbances for the same conditions. The scale of the isobaric density perturbations is bounded between 0.176 and -0.118 , the maximum and minimum value for $\bar{\rho}_{e}$ in the domain $k_{y} x>0$.

\section{APPENDIX B: DETAILS OF THE DIRECT NUMERICAL SIMULATIONS}

The absence of an accessible analytical theory that includes nonlinear effects and situations where the characteristic length of turbulence is comparable to the detonation thickness calls for other techniques, such as accurate numerical simulations capable of capturing these effects. With this aim, a set of computations, with a bandwidth-optimized WENO scheme that was presented in [33], has been performed. 

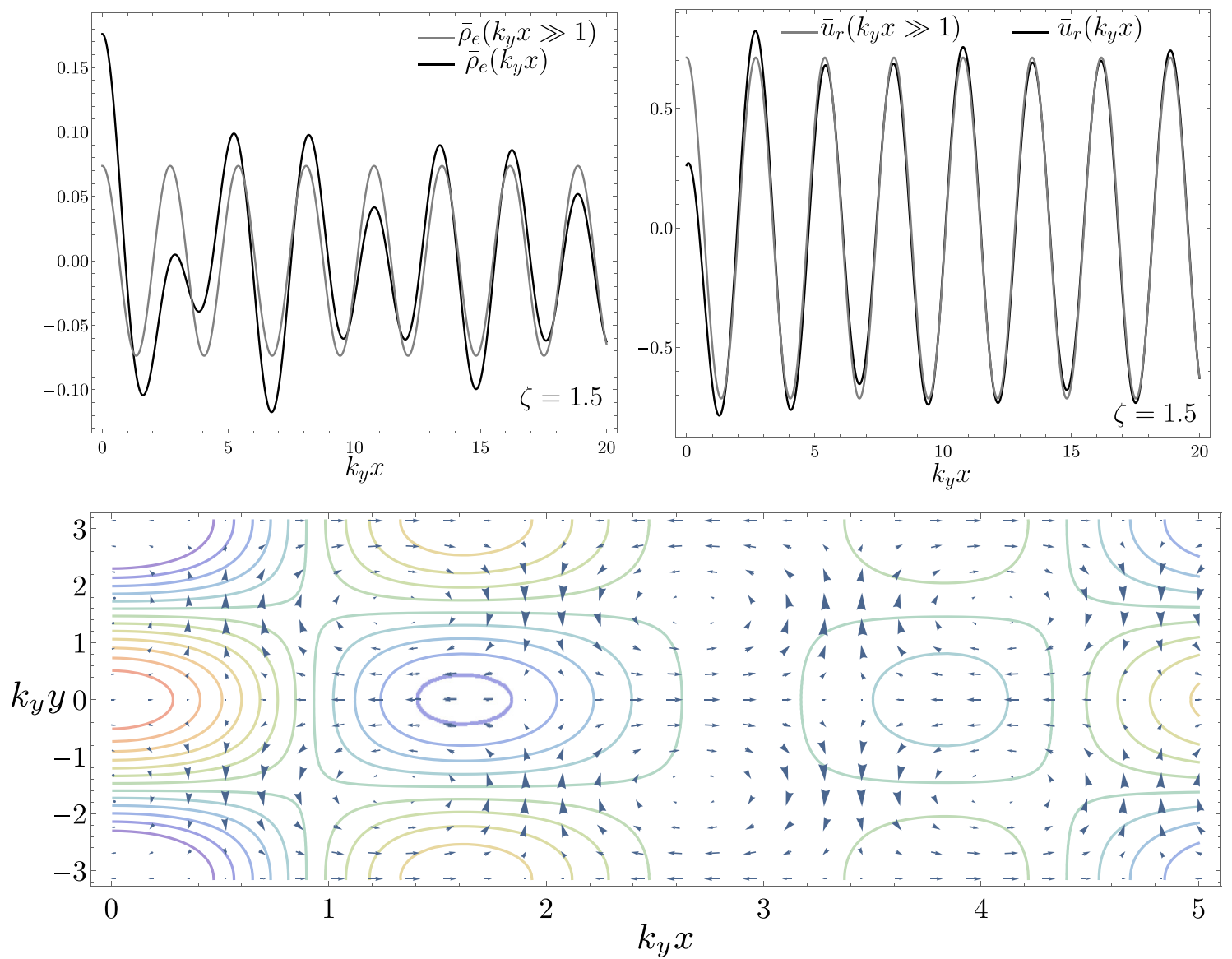

FIG. 18. Upper panel: entropic-density and longitudinal rotational-velocity perturbations as a function of $k_{y} x$ for $M_{0}=1.5 M_{\mathrm{CJ}}, q=1$, $\gamma=1.4$, and $\zeta=1.5$. Lower panel: two-dimensional vector field plot for rotational-velocity perturbations superposed to the isocontours of entropic-density disturbances for a detonation wave traveling at the same conditions.

The DNS algorithm computes the governing equations for compressible reactive flows, including the three-dimensional Navier-Stokes equations, the conservation equations of chemical species $Y$, the conservation equation of the total energy $E_{t}=p /\left[\gamma(\gamma-1) M_{0}^{2}\right]+\rho\left[u^{2} / 2+Q(1-Y)\right]$, and the perfect-gas equation of state:

$$
\begin{gathered}
\frac{\partial \rho}{\partial t}+\frac{\partial\left(\rho u_{i}\right)}{\partial x_{i}}=0, \\
\frac{\partial\left(\rho u_{i}\right)}{\partial t}+\frac{\partial\left(\rho u_{i} u_{j}\right)}{\partial x_{j}}=-\frac{\partial\left[p /\left(\gamma M_{0}^{2}\right) \delta_{i j}\right]}{\partial x_{j}}+\frac{\partial}{\partial x_{j}}\left[\frac{\mu}{\operatorname{Re}}\left(\frac{\partial u_{i}}{\partial x_{j}}+\frac{\partial u_{j}}{\partial x_{i}}-\frac{2}{3} \frac{\partial u_{k}}{\partial x_{k}} \delta_{i j}\right)\right], \quad i=1,2,3 \\
\frac{\partial(\rho Y)}{\partial t}+\frac{\partial\left(\rho u_{j} Y\right)}{\partial x_{j}}=\frac{\partial}{\partial x_{j}}\left(\frac{\mu}{\operatorname{RePrLe}} \frac{\partial Y}{\partial x_{j}}\right)-K \rho Y e^{-T_{a} / T}, \\
\frac{\partial E_{t}}{\partial t}+\frac{\partial\left(u_{j} E_{t}\right)}{\partial x_{j}}=-\frac{\partial u_{j}\left[p /\left(\gamma M_{0}^{2}\right)\right]}{\partial x_{j}}+\frac{\partial}{\partial x_{j}}\left[\frac{u_{i} \mu}{\operatorname{Re}}\left(\frac{\partial u_{i}}{\partial x_{j}}+\frac{\partial u_{j}}{\partial x_{i}}-\frac{2}{3} \frac{\partial u_{k}}{\partial x_{k}} \delta_{i j}\right)\right]+\frac{\partial}{\partial x_{j}}\left[\frac{\mu}{\operatorname{RePr}(\gamma-1) M_{0}^{2}} \frac{\partial T}{\partial x_{j}}\right], \\
p=\rho R_{g} T,
\end{gathered}
$$

where the dimensionless heat release per unit mass of reactant burnt is $q=50$. A power-law approximation is used for the viscosity-coefficient dependence with temperature variation $\mu=\left(T / T_{0}\right)^{0.7}$. The reference Reynolds number is $\mathrm{Re}=$ $\left(\rho_{0} u_{t} l_{t}\right) / \mu_{0}=498.8$, which has been determined with $\mu_{0}=$
$2 \times 10^{-5} \mathrm{~kg} /(\mathrm{m} \mathrm{s})$, the length scale $l_{t}=1.154 \times 10^{-4} \mathrm{~m}$, and velocity $u_{t}=86.65 \mathrm{~m} / \mathrm{s}$, values to be employed in the nondimensionalization of the Navier-Stokes equations integrated numerically. The Reynolds number associated to the Taylor microscale, computed with the root mean square of the 
preshock velocity fluctuations and $\lambda_{t}=3.49 \times 10^{-5} \mathrm{~m}$ yields $\operatorname{Re}_{t}=37.8$. The Prandtl number is set to $\operatorname{Pr}=\left(\mu_{0} c_{p}\right) / \kappa=0.7$ and Lewis number Le $=\kappa /\left(\rho_{0} c_{p} D\right)=1$. As the Reynolds numbers is significantly high in detonation-type flows, the effect of species and temperature diffusion is expected to show a subdominant effect in the reaction region. For this reason, these dimensionless numbers are kept constant in the four scenarios considered. As in (9), an Arrhenius-type one-step global chemical reaction has been considered and the reference frame is set to move with the steady detonation wave.

Periodic boundary conditions are imposed in all three directions to produce the isotropic turbulence feed, enclosing a $192^{3}$ mesh discretization of a cubic domain of size $(2 \pi)^{3}$. The dimensional computation domain $\left(2 \pi l_{t}\right)^{3}$ is scaled with the upstream Taylor microscale $\lambda_{t}$ to provide a volume $(6.61 \pi)^{3}$ in dimensionless quantities. In consonance with the isolated-detonation boundary condition employed in LIA, an equivalent nonreflective boundary condition is used in the numerical simulations to prescribe the outflow boundary and avoid acoustic reflections in the subsonic flow.

The upstream turbulence employed to feed the flow computations is described by statistical values of the quantities that characterize the flow field, which in turn may depend on both position and time. Nevertheless, the turbulent flow upstream is assumed to be isotropic and homogeneous in agreement with the LIA developed in previous sections. With the same spirit as in [33], a divergence-free velocity field with constant pressure is used as an initial condition for the simulation decaying turbulence characterized by the energy spectrum [45]

$$
E(k)=A k^{4} \exp \left(-\frac{2 k^{2}}{k_{0}^{2}}\right)
$$

where $k$ stands for the wave number, $k_{0}$ is the wave number at which the spectrum peaks, and $A$ is the free constant chosen to tune a specified initial kinetic energy. In particular, the values of $A=4.434 \times 10^{-3}$ and $k_{0}=4$ have been selected in the performed simulations. The homogeneous isotropic turbulence is characterized by a negative skewness of the velocity derivative

$$
S_{i}=-\frac{\left\langle\left(\partial u_{i}^{\prime} / \partial x_{i}\right)^{3}\right\rangle}{\left\langle\left[\left(\partial u_{i}^{\prime} / \partial x_{i}\right)^{2}\right]^{3 / 2}\right\rangle},
$$

which represents the rate of production of vorticity through vortex stretching. Time decay is stopped after the fluctuating fields are stabilized to a mean velocity derivative skewness $S=\left(S_{1}+S_{2}+S_{3}\right) / 3=-0.5$, that makes them reasonably representative of real turbulence. The Kolmogorov length scale $\eta=\left(\left\langle\mu_{0} / \rho_{0}\right\rangle^{3} / \epsilon\right)^{1 / 4}$, with $\epsilon$ representing the rate of dissipation of the kinetic energy, must be resolved with a sufficiently fine spatial mesh where $\Delta x / \eta$ is small enough or as commonly defined, where the dissipation spectra are extremely small $k \eta \gtrsim 1.5$, so that the simulations performed with $k_{\max } \eta=4.69$ adequately serve to describe isotropic turbulence upstream [46]. It is clear that $k_{\max } \eta$ changes behind the detonation wave, yielding the most restrictive condition $k_{\max } \eta=1.53$ in the unstable case.

In particular, the simulations cover distinguished scenarios that include the interaction with a nonreactive shock wave, case NR, as a reference canonical situation and three different reactive cases, whose computational parameters are collected in Table I. The case RU describes the effect on the turbulent flow of a reactive-unstable detonation wave, by considering a relatively high activation energy $E_{a}=50 R_{g} T_{0}$ and a relatively low overdrive factor $M_{0}=1.5 M_{\mathrm{CJ}}$, when the upstream perturbation length scale is of the same order of the detonation thickness $\ell / \lambda_{t} \sim 1$, with $\ell$ being the half-reaction length provided by (10) in steady laminar conditions. In addition, two stable scenarios are tested by varying the reaction parameter to a lower temperature-sensitive case, that is, considering lower activation energy instead, $E_{a}=10 R_{g} T_{0}$. Case RS1 is set to represent situations where $\ell / \lambda_{t} \sim 1$, while case RS2 computes thinner detonation waves $\ell / \lambda_{t} \sim 0.1$ by increasing the value of the frequency factor of the reaction rate $B$. Then, the effect of the induction length in the turbulent kinetic energy can be studied independently, with the global detonation properties kept invariant. Both cases RS1 and RS2 have been previously proven stable with perturbation-free simulations.
[1] H. S. Ribner, NACA Report No. 1164, 1954.

[2] H. S. Ribner, NACA Report No. 1233, 1955.

[3] H. S. Ribner, AIAA J. 25, 436 (1987).

[4] F. E. Marble et al., Turb. React. Flows 40, 932 (1989).

[5] S. Menon, 27th Aerospace Sciences Meeting (AIAA, Reston, VA, 1989).

[6] E. J. Gutmark et al., Annu. Rev. Fluid Mech. 27, 375 (1995).

[7] K. Kailasanath, AIAA J. 38, 1698 (2000).

[8] G. Fusina, Ph.D. thesis, University of Toronto, 2003.

[9] B. T. Draine and C. F. McKee, Annu. Rev. Astron. Astrophys. 31, 373 (1993).

[10] E. Abdikamalov et al., Mon. Not. R. Astron. Soc. 461, 3864 (2016).

[11] B. Müller et al., Astrophys. J. 833, 124 (2016).

[12] L. Jacquin et al., Phys. Fluids A 5, 2539 (1993).

[13] S. Barre et al., AIAA J. 34, 968 (1996).
[14] S. Lee et al., J. Fluids Mech. 340, 225 (1997).

[15] K. Mahesh et al., J. Fluids Mech. 334, 353 (1997).

[16] E. Garnier et al., Comput. Fluids 31, 245 (2002).

[17] J. Larsson and S. K. Lele, Phys. Fluids 21, 126101 (2009).

[18] J. G. Wouchuk, C. Huete Ruiz de Lira, and A. L. Velikovich, Phys. Rev. E 79, 066315 (2009).

[19] V. K. Veera and K. Sinha, Phys. Fluids 21, 025101 (2009).

[20] F. Génin and S. Menon, Comput. Fluids 39, 800 (2010).

[21] J. Griffond and O. Soulard, Phys. Fluids 24, 115108 (2012).

[22] J. Larsson et al., J. Fluids Mech. 717, 293 (2013).

[23] J. Ryu and D. Livescu, J. Fluids Mech. 756, R1 (2014).

[24] D. Livescu and J. Ryu, Shock Waves 26, 241 (2015).

[25] P. Rajea and K. Sinha, Comput. Fluids 136, 35 (2016).

[26] R. Quadros et al., J. Fluids Mech. 796, 113 (2016).

[27] T. L. Jackson et al., Phys. Fluids A 2, 1260 (1990).

[28] T. L. Jackson et al., Phys. Fluids A 5, 745 (1993). 
[29] L. Massa and F. K. Lu, Combust. Theory Modell. 15, 347 (2011).

[30] L. Massa et al., Combust. Flame 158, 1788 (2011).

[31] C. Huete et al., Phys. Fluids 25, 076105 (2013).

[32] C. Huete et al., Phys. Fluids 26, 116101 (2014).

[33] T. Jin et al., AIAA J. 54, 419 (2016).

[34] C. Huete et al., J. Fluids Mech. 784, 74 (2015).

[35] A. L. Velikovich, C. Huete, and J. G. Wouchuk, Phys. Rev. E 85, 016301 (2012).

[36] R. Daou and P. Clavin, J. Fluids Mech. 482, 181 (2003).

[37] P. Clavin and F. A. Williams, Philos. Trans. R. Soc. London A 370, 597 (2012).

[38] Y. B. Zel'dovich, Zh. Eksp. Teor. Fiz. 10, 542 (1940).
[39] J. von Neumann, Theory of detonation waves, Prog. Rep. No. 238 (Report 549, O.S.R.D, National Defense Research Committee Div. B, 1942).

[40] W. Doring, Ann. Phys. (NY) 43, 421 (1943).

[41] V. P. Korobeinikov, Annu. Rev. Fluid Mech. 3, 317 (1971).

[42] B. T. Chu and L. S. G. Kovásznay, J. Fluids Mech. 3, 494 (1958).

[43] G. K. Batchelor, The Theory of Homogeneous Turbulence (Cambridge University Press, Cambridge, England, 1953).

[44] G. Fraley, Phys. Fluids 29, 376 (1986).

[45] R. Samtaney et al., Phys. Fluids 13, 1415 (2001).

[46] S. B. Pope, Turbulent Flows (Cambridge University Press, Cambridge, UK, 2000). 